\title{
Lorentz and $C P T$ tests with charge-to-mass ratio comparisons in Penning traps
}

\author{
Yunhua Ding $\odot^{*}$ and Mohammad Farhan Rawnak $\bullet$ \\ Department of Physics, Gettysburg College, Gettysburg, Pennsylvania 17325, USA
}

(Received 27 July 2020; accepted 17 August 2020; published 9 September 2020)

\begin{abstract}
Applications of the general theory of quantum electrodynamics with Lorentz- and CPT-violating operators of mass dimensions up to six are presented to Penning-trap experiments comparing charge-tomass ratios between particles and antiparticles. Perturbation theory is used to derive Lorentz- and CPTviolating contributions to the energy levels and cyclotron frequencies of confined particles and antiparticles. We show that whether the experimental interpreted quantity $(|q| / m)_{\bar{w}} /(|q| / m)_{w}-1$ is a clean measure of a $C P T$ test depends on the context of the relevant theory. Existing experimental results of charge-to-mass ratio comparisons are used to obtain first-time constraints on 69 coefficients for Lorentz and $C P T$ violation.
\end{abstract}

DOI: 10.1103/PhysRevD.102.056009

\section{INTRODUCTION}

Invariance under Lorentz transformations is one of the fundamental symmetries of both general relativity and the Standard Model of particle physics. However, tiny violations of Lorentz invariance could naturally emerge via spontaneous symmetry breaking in a fundamental theory unifying gravity with quantum physics, such as string theory [1]. In realistic effective field theory any violation of $C P T$ symmetry, the invariance under the combined transformation of charge conjugation $\mathrm{C}$, parity inversion $\mathrm{P}$, and time reversal $\mathrm{T}$, is accompanied by Lorentz violation $[2,3]$. It follows that testing Lorentz symmetry includes $C P T$ tests as well. Motivated by this, many high-precision experiments in various subfields of physics have been performed to search for a variety of Lorentz- and $C P T$ violating signals [4].

Testing Lorentz and $C P T$ symmetry requires either the study of the effects of a physical system under rotations or boosts, or comparing the fundamental properties of a particle such as lifetime, charge-to-mass ratio, and $g$ factor to these of its antiparticle. Among the high-precision tests of Lorentz and CPT invariances, the Penning trap is of particular interest, as it provides a stable confinement of a particle or an antiparticle, permitting highly precise measurements and comparisons of its properties. Meanwhile, the Earth provides a natural rotating and boosting frame to

\footnotetext{
*yding@gettysburg.edu
}

Published by the American Physical Society under the terms of the Creative Commons Attribution 4.0 International license. Further distribution of this work must maintain attribution to the author(s) and the published article's title, journal citation, and DOI. Funded by SCOAP ${ }^{3}$. study these properties under Lorentz transformations. Impressive sensitivities have been achieved by Penningtrap experiments. For example, the proton and antiproton charge-to-mass ratios were compared to parts per trillion [5]. For the $g$ factors of electrons and positrons, as well as these of protons and antiprotons, the precision achieved was at parts per billion [6,7]. The prospects of testing Lorentz and $C P T$ symmetry in Penning-trap experiments measuring the $g$ factors of particles and antiparticles were addressed in Ref. [8]. To extend that work, we focus in this paper on searches for Lorentz and $C P T$ violation using the charge-to-mass ratio comparisons between particles and antiparticles confined in a Penning trap.

The comprehensive framework to study Lorentz and $C P T$ violation in the context of effective field theory is known as the Standard-Model Extension (SME) [2,9], which is constructed from the action of general relativity and the Standard Model by adding all possible Lorentzviolating terms. Each of these terms is formed from a coordinate-independent contraction of a Lorentz-violating operator with a corresponding controlling coefficient. The subset of the SME containing operators of powercounting renormalizable mass dimension $d \leq 4$ is called the minimal SME, while the nonminimal SME restricts attention to operators of mass dimensions $d>4$ and is assumed to produce higher-order corrections to conventional physics.

Both the minimal and nonminimal SME can produce various Lorentz- and $C P T$-violating effects in Penning-trap experiments, including those measuring the $g$ factor and charge-to-mass ratio of a confined particle or antiparticle [5-7,10-12]. These effects include shifts in the cyclotron and anomaly frequencies that can depend on sidereal time and also differ between particles and antiparticles. The 
original theoretical work using the minimal SME to study Lorentz and $C P T$ violation was given in Refs. [13,14]. It was recently extended to the nonminimal SME by including Lorentz- and $C P T$-violating operators of dimensions up to six, together with applications to Penning-trap experiments comparing the $g$ factors between a particle and an antiparticle [8]. The searches for the effects arising from sidereal variations in these experiments have also been discussed [15-17].

However, no treatment on the nonminimal SME effects in Penning-trap experiments comparing charge-to-mass ratios exists in the literature to date. Addressing these nonminimal effects is of significance as it can reveal additional measurable Lorentz- and $C P T$-violating signals due to the interactions of the particle or antiparticle with the electromagnetic fields in the trap. More generally, studying the nonminimal SME sector can provide crucial insights to many aspects of Lorentz and $C P T$ violation, such as noncommutative Lorentz-violating quantum electrodynamics $[18,19]$, Lorentz-violating models in supersymmetry [20], or foundational issues including causality and stability [21] and the underlying pseudo-RiemannFinsler geometry [22].

In this work, we address this gap by studying the nonminimal SME effects arising from particle and antiparticle charge-to-mass ratio measurements in Penning traps. The theory of Lorentz- and $C P T$-violating electrodynamics with operators of mass dimensions up to six developed in Ref. [8] provides a partial guide to investigate these effects. Applying perturbation theory we derive the leading-order contributions due to Lorentz and CPT violation to cyclotron frequencies and then relate them to charge-to-mass ratio comparisons. We also address the question of whether a comparison of the experimental interpreted charge-to-mass ratios between particles and antiparticles is a clean $C P T$ test and conclude that it depends on the context of the relevant theory. Taking published results including the sidereal studies from Penning-trap experiments, we extract first-time constraints on 69 SME coefficients. The results obtained in this work are complementary to existing ones from comparisons of the $g$ factors between particles and antiparticles in Penningtrap experiments $[8,16]$, the studies of the anomalous magnetic moment of muons confined in a storage ring $[23,24]$, the spectroscopic investigations of hydrogen, antihydrogen, and other related systems [25], and clockcomparison experiments [26].

This work is organized as follows. In Sec. II, we revisit the theory of quantum electrodynamics with Lorentz- and $C P T$-violating operators of mass dimensions up to six and derive the perturbative Hamiltonian at leading order in Lorentz and CPT violation. We next turn in Sec. III the applications to Penning-trap experiments. The dominant energy shifts due to Lorentz and $C P T$ violation of a confined particle or antiparticle are given in Sec. III A, followed in Sec. III B by the corresponding cyclotron frequency shifts. We address in Sec. IIIC the general transformation of the coefficients for Lorentz violation between different frames. This leads to a discussion in Sec. III D of possible measurable Lorentz- and $C P T$ violating signals in Penning-trap experiments comparing charge-to-mass ratios between particles and antiparticles. In Sec. IIIE, we use published experimental results to extract first-time constraints on 69 SME coefficients and summarize them in Table II. Finally, we given in Sec. IV the summary of this work. Three Appendixes are given at the end of the paper for the reader' convenience. In Appendix A, we reproduce the full Lagrange density of quantum electrodynamics with Lorentz- and $C P T$ violating operators of mass dimensions $d \leq 6$. The explicit calculation result of the perturbative energy shifts is given in Appendix B, followed in Appendix $\mathrm{C}$ by the transformation results for the related coefficients for Lorentz violation.

Throughout the paper, we follow the notation used in Refs. $[8,27]$, unless otherwise specified. In particular, we adopt natural units with $\hbar=c=1$ and express mass units in $\mathrm{GeV}$.

\section{THEORY}

In this section, we focus on the theory by revisiting the Lagrange density of Lorentz-violating spinor electrodynamics with operators of mass dimensions up to six [8] and deriving the related perturbative Hamiltonian at leading order in Lorentz and $C P T$ violation.

\section{A. Lagrange density}

In the framework of the SME, the general Lorentzviolating Lagrange density that preserves $U(1)$ gauge invariance for a single Dirac fermion field $\psi$ of charge $q$ and mass $m_{\psi}$ coupled to an electromagnetic field $A_{\mu}$ is given by

$$
\mathcal{L}_{\psi}=\mathcal{L}_{0}+\frac{1}{2} \bar{\psi} \hat{\mathcal{Q}} \psi+\text { H.c. }
$$

where $\mathcal{L}_{0}=\frac{1}{2} \bar{\psi}\left(\gamma^{\mu} i D_{\mu}-m_{\psi}\right) \psi+$ H.c. is the conventional Lorentz-invariant QED Lagrange density, with $i D_{\mu}$ being the covariant derivative from the minimal coupling $i D_{\mu} \equiv i \partial_{\mu}-q A_{\mu}$ and H.c. denoting Hermitian conjugate. $\hat{\mathcal{Q}}$ is a general $4 \times 4$ Lorentz-violating operator involving the covariant derivative $i D_{\mu}$ and the antisymmetric electromagnetic field tensor $F_{\mu \nu} \equiv \partial_{\mu} A_{\nu}-\partial_{\nu} A_{\mu}$. From the Hermiticity of the Lagrange density (1), $\hat{\mathcal{Q}}$ satisfies $\hat{\mathcal{Q}}=\gamma_{0} \hat{\mathcal{Q}}^{\dagger} \gamma_{0}$. The spin content of $\hat{\mathcal{Q}}$ can be shown by expanding it in the basis of the 16 Dirac matrices,

$$
\hat{\mathcal{Q}}=\hat{\mathcal{S}}+i \hat{\mathcal{P}} \gamma_{5}+\hat{\mathcal{V}}^{\mu} \gamma_{\mu}+\hat{\mathcal{A}}^{\mu} \gamma_{5} \gamma_{\mu}+\frac{1}{2} \hat{\mathcal{T}}^{\mu \nu} \sigma_{\mu \nu}
$$


where the 16 operators $\left\{\hat{\mathcal{S}}, \hat{\mathcal{P}}, \hat{\mathcal{V}}^{\mu}, \hat{\mathcal{A}}^{\mu}, \hat{\mathcal{T}}^{\mu \nu}\right\}$ are Diracscalar functions of mass dimension one formed from the contraction of coefficients for Lorentz violation and operators including $i D_{\mu}$ and $F_{\mu \nu}$ in general. For example, one of the dimension-five terms in $\hat{\mathcal{A}}^{\mu}$ takes the form $-\frac{1}{2} b_{f}^{(5) \mu \alpha \beta} F_{\alpha \beta}$, where $b_{f}^{(5) \mu \alpha \beta}$ is the controlling coefficient for Lorentz violation. As shown in Ref. [8], this term can produce both Lorentz- and $C P T$-violating effects in experiments measuring the magnetic moment of a particle or an antiparticle with a Penning trap.

The explicit form of the Lagrange density (1) at arbitrary mass dimension in the free-fermion limit $A_{\mu}=0$ has been studied in Ref. [27]. For a Dirac fermion interacting with a nonzero $A_{\mu}$, its expression for a mass dimension $d \leq 6$ was constructed in Ref. [8], where a set of novel $F$-type coefficients for Lorentz violation associated with $F_{\mu \nu}$ was discussed. An extension to arbitrary mass dimension for the interacting case was recently given by Ref. [28]. For other SME sectors, a similar analysis of the quadratic terms in the photon sector at an arbitrary mass dimension has been presented in Ref. [29], as well as extensions to the neutrino sector [30], and the gravity sector [31]. Since the Lagrange density constructed in Ref. [8] serves as the theoretical basis of the present work, for convenience we reproduce these terms in Appendix A.

\section{B. Perturbative Hamiltonian}

Given that no Lorentz- and $C P T$-violating signals have been observed in experiments to date, any such possible symmetry-violating effects must be tiny. Therefore, the contributions from the Lorentz-violating operator $\hat{\mathcal{Q}}$ to the Hamiltonian related to the Lagrange density (1) can be treated as perturbative. To derive the explicit expression of the perturbative Hamiltonian $\delta \mathcal{H}$, we start from the modified Dirac equation in the momentum space,

$$
\left(p \cdot \gamma-m_{\psi}+\hat{\mathcal{Q}}\right) \psi=0,
$$

where $p_{\mu} \equiv i D_{\mu} \equiv\left(i \partial_{\mu}-q A_{\mu}\right)$. The exact Hamiltonian $\mathcal{H}$ can then be defined from Eq. (3) via

$$
\mathcal{H} \psi \equiv p^{0} \psi=\gamma_{0}\left(\boldsymbol{p} \cdot \boldsymbol{\gamma}+m_{\psi}-\hat{\mathcal{Q}}\right) \psi,
$$

where $p^{0}$ is the exact energy of the physical system including Lorentz violation. Separating the exact Hamiltonian $\mathcal{H}$ into the sum of the conventional Hamiltonian $\mathcal{H}_{0}$ and the perturbative part $\delta \mathcal{H}$ due to Lorentz and $C P T$ violation, we identify the exact perturbative Hamiltonian to be $\delta \mathcal{H}=-\gamma_{0} \hat{\mathcal{Q}}$.

It is challenging to construct the perturbative Hamiltonian $\delta \mathcal{H}$ directly since the Lorentz-violating operator $\hat{\mathcal{Q}}$ in general contains powers of $p_{0}$ and thus includes the perturbative Hamiltonian $\mathcal{H}$ itself. This implies that the standard procedure cannot be adopted to obtain a Dirac Hamiltonian operator generating time translations on the wave function. For certain cases of $\hat{\mathcal{Q}}$, a field redefinition at the level of the Lagrange density can be performed to overcome this difficulty by removing the additional time derivatives [14]. However, at the leading order in Lorentz violation, a more general procedure, first presented in Ref. [30], can be adopted by noticing that any contributions to $\delta \mathcal{H}$ due to the exact Hamiltonian $\mathcal{H}$ are at second or higher orders in Lorentz violation. Therefore, to obtain the leading-order effects, the perturbative Hamiltonian $\delta \mathcal{H}$ can be evaluated using the unperturbative energy $E_{0}$ for $p^{0}$,

$$
\delta \mathcal{H} \approx-\left.\gamma_{0} \hat{\mathcal{Q}}\right|_{p^{0} \rightarrow E_{0}},
$$

where $E_{0}$ can be derived by solving the relevant conventional Dirac equation for the physical system.

\section{APPLICATION TO THE PENNING TRAP}

In this section, we apply the above theory to experiments involving Penning traps. Using perturbation theory we derive the energy shifts due to Lorentz and $C P T$ violation of particles and antiparticles confined in a Penning tap. Then we obtain the dominant shifts in their cyclotron frequencies, followed by a discussion of general frame changes to study the transformation under rotations. This leads to investigations of possible measurable signals in Penning-trap experiments comparing the charge-tomass ratios between particles and antiparticles, including a discussion of the $C P T$ test. By identifying the relations between experimental measured quantities and coefficients for Lorentz violation, we obtain first-time constraints on 69 SME coefficients from published Penning-trap results.

\section{A. Energy shifts}

For precision experiments involving particles or antiparticles confined in a Penning trap, the relevant experimental observables of interest are frequencies, which are the energy differences between energy levels. To obtain the shifts in the energy levels of a confined particle due to Lorentz and $C P T$ violation, we apply perturbation theory,

$$
\delta E_{n, \pm}=\left\langle\chi_{n, \pm}|\delta \mathcal{H}| \chi_{n, \pm}\right\rangle,
$$

where $\delta \mathcal{H}$ is the perturbative Hamiltonian given by (5), $\chi_{n, \pm}$ denote the unperturbative four-component stationary eigenstates of level number $n$ and spin \pm for the positiveenergy fermion, and $\delta E_{n, \pm}$ are the corresponding perturbative energy shifts due to Lorentz and $C P T$ violation.

In Penning-trap experiments, the dominant effects in the unperturbative energy spectrum arise from the interaction of the confined particle or antiparticle with the constant 
magnetic field of the trap. The quadrupole electric field, which varies with the position to provide the axial confinement, generates weaker effects suppressed by a factor of $E / B$. In a typical trap with $E \approx 20 \mathrm{kV} / \mathrm{m}$ and $B \approx 5 \mathrm{~T}$, this ratio is about $10^{-5}$ in natural units. Therefore, we start the theoretical analysis with an idealized scenario where a relativistic quantum fermion moves in a uniform magnetic field only. The unperturbative fermion eigenstates $\chi_{n, \pm}$ in the absence of Lorentz and CPT violation can be obtained by solving the conventional Dirac equation with the minimal coupling for a spin-1/2 fermion of mass $m$ and charge $q \equiv \sigma|q|$ in a constant magnetic field. For calculation definiteness, we fix the gauge with $A^{\mu}=$ $\left(0, x_{2} B, 0,0\right)=\left(0,-x^{2} B, 0,0\right)$ so that the magnetic field is $\boldsymbol{B}=B \hat{x}_{3}$, pointing the positive $x^{3}$ axis in the apparatus frame.

After some calculation, we present in Appendix B the explicit result of the energy shifts $\delta E_{n, \pm}^{w}$ for a fermion of species $w$ and charge sign $\sigma$ in a magnetic field $\boldsymbol{B}=B \hat{x}_{3}$ due to Lorentz-violating operators appearing in $\mathcal{L}^{(3)}, \mathcal{L}^{(4)}$, $\mathcal{L}_{D}^{(5)}$, and $\mathcal{L}_{D}^{(6)}$, given in Appendix A. The additional energy shift contributions from operators in $\mathcal{L}_{F}^{(5)}$ and $\mathcal{L}_{F}^{(6)}$ can be obtained via substitutions listed in (40) in Ref. [8], while terms in $\mathcal{L}_{\partial F}^{(6)}$ produce no energy shift contributions as $\partial_{\alpha} F_{\beta \gamma}=0$ for a uniform magnetic field in a Penning trap. In obtaining the result (B1), we note that the axial motion of the confined particle or antiparticle in a Penning trap is purely induced by the electric field, this means terms involving one or more powers of the Landau momentum $p_{3}$ appearing in the energy shift calculation are also suppressed by one or more powers of the ratio $E / B$. Therefore, we disregard such terms in result (B1) to obtain the leading-order contributions. Note also that the unperturbed positive eigenenergies in result (B1) take the form $E_{n, \pm 1}^{w}=\sqrt{m_{w}^{2}+(2 n+1 \mp \sigma)|q B|}$.

As shown in Appendix B, the full energy shifts (B1) depend on several variables, including the charge sign $\sigma$ of the particle, the spin orientation, and the level number $n$. The dependence on the direction of the magnetic field is reflected by the indices of the coefficients for Lorentz violation as the calculations are performed in the apparatus frame with the magnetic field along the positive $x^{3}$ axis. The magnitude dependence is evident from terms involving powers of $|q B|$. Since in a typical Penning-trap experiment a particle with $1 e$ charge in a magnetic field of $B \approx 5 \mathrm{~T}$ corresponds to $|q B| \approx 10^{-16} \mathrm{GeV}^{2}$ in natural units, to obtain the leading-order contributions due to the magnetic field we can expand terms containing $E_{n, \pm 1}^{w}$ in Taylor series of $|q B|$ and keep only up to the linear terms in $|q B|$ in the result.

With the above approximations and including also the contributions from operators in $\mathcal{L}_{F}^{(5)}$ and $\mathcal{L}_{F}^{(6)}$, we can rewrite the perturbative energy shifts (B1) in the form,

$$
\begin{aligned}
\delta E_{n, \pm 1}^{w}= & \tilde{\alpha}_{w}^{0} \mp \sigma \tilde{\beta}_{w}^{3}-\tilde{m}_{F, w}^{3} \pm \sigma \tilde{b}_{F, w}^{33} B \\
& +\left( \pm \sigma \tilde{b}_{w}^{33}-m_{w}\left[\tilde{c}_{w}^{00}+\left(\tilde{c}_{w}^{11}+\tilde{c}_{w}^{22}\right)_{s}\right]\right) \frac{(2 n+1 \mp \sigma)|q B|}{2 m_{w}^{2}} \\
& +\left(\mp \sigma\left(\tilde{b}_{w}^{311}+\tilde{b}_{w}^{322}\right)-\frac{1}{m_{w}}\left(\tilde{c}_{w}^{11}+\tilde{c}_{w}^{22}\right)_{s}\right) \frac{(2 n+1)|q B|}{2}
\end{aligned}
$$

where the various tilde coefficients are defined by

$$
\begin{aligned}
\tilde{a}_{w}^{0}= & a_{w}^{0}-m_{w} c_{w}^{00}-m_{w} e_{w}^{0}+m_{w}^{2} m_{w}^{(5) 00} \\
& +m_{w}^{2} a_{w}^{(5) 000}-m_{w}^{3} c_{w}^{(6) 0000}-m_{w}^{3} e_{w}^{(6) 000} \\
\tilde{b}_{w}^{3}= & b_{w}^{3}+H_{w}^{12}-m_{w} d_{w}^{30}-m_{w} g_{w}^{120}+m_{w}^{2} b_{w}^{(5) 300} \\
& +m_{w}^{2} H_{w}^{(5) 1200}-m_{w}^{3} d_{w}^{(6) 3000}-m_{w}^{3} g_{w}^{(6) 12000} \\
\tilde{m}_{F, w}^{3}= & m_{F, w}^{(5) 12}+a_{F, w}^{(5) 012}-m_{w} c_{F, w}^{(6) 0012}-m_{w} e_{F, w}^{(6) 012} \\
\tilde{b}_{F, w}^{33}= & b_{F, w}^{(5) 312}+H_{F, w}^{(5) 1212}-m_{w} d_{F, w}^{(6) 3012}-m_{w} g_{F, w}^{(6) 12012} \\
\tilde{b}_{w}^{\prime 3}= & b_{w}^{3}+m_{w}\left(g_{w}^{110}-g_{w}^{012}+g_{w}^{021}\right)-m_{w}^{2} b_{w}^{(5) 300} \\
& -2 m_{w}^{2}\left(H_{w}^{(5) 1200}-H_{w}^{(5) 0102}+H_{w}^{(5) 0201}\right) \\
& +2 m_{w}^{3} d_{w}^{(6) 3000}+3 m_{w}^{3}\left(g_{w}^{(6) 12000}-g_{w}^{(6) 01002}+g_{w}^{(6) 02001}\right), \\
\tilde{c}_{w}^{00}= & c_{w}^{00}-m_{w} m_{w}^{(5) 00}-2 m_{w} a_{w}^{(5) 000} \\
& +3 m_{w}^{2} c_{w}^{(6) 0000}+2 m_{w}^{2} e_{w}^{(6) 000},
\end{aligned}
$$

and the " $11+22$ " types of tilde coefficients are defined by

$$
\begin{aligned}
\left(\tilde{c}_{w}^{j j}\right)_{s}= & c_{w}^{j j}-2 m_{w} a_{w}^{(5) j 0 j}+3 m_{w}^{2} c_{w}^{(6) j 00 j}, \\
\left(\tilde{c}_{w}^{j j}\right)_{s}= & -m_{w} a_{w}^{(5) 0 j j}-m_{w} m_{w}^{(5) j j} \\
& +3 m_{w}^{2} c_{w}^{(6) 00 j j}+3 m_{w}^{2} e_{w}^{(6) 0 j j} \\
\tilde{b}_{w}^{3 j j}= & b_{w}^{(5) 3 j j}+H_{w}^{(5) 12 j j} \\
& -3 m_{w} d_{w}^{(6) 30 j j}-3 m_{w} g_{w}^{(6) 120 j j}
\end{aligned}
$$

with $j$ taking values of 1 and 2 only. The subscripts $s$ and $\vec{s}$ in the above $\tilde{c}_{w}^{j j}$ tilde coefficients specify the fact that $\left(\tilde{c}_{w}^{j j}\right)_{s}$ produce both spin-independent and spin-dependent energy shift contributions, while $\left(\tilde{c}_{w}^{j j}\right)_{s}$ give only spin-independent ones, which is evident from the corresponding proportional factors $2 n+1 \mp \sigma$ and $2 n+1$ in the result (7). We note that the energy shift contributions from tilde coefficients $\tilde{a}_{w}^{0}, \tilde{b}_{w}^{3}, \tilde{m}_{F, w}^{3}$, and $\tilde{b}_{F, w}^{33}$ are independent of the level number $n$. Expression (7) extends the energy shift result obtained in Ref. [8] by including terms linear in $|q B|$. These terms can lead to nonzero contributions to the cyclotron frequencies, as will be shown in the next subsection.

The corresponding shifts in the antifermion energy levels due to Lorentz and $C P T$ violation are given by 


$$
\delta E_{n, \pm}^{c}=\left\langle\chi_{n, \pm}^{c}\left|\delta \mathcal{H}^{c}\right| \chi_{n, \pm}^{c}\right\rangle
$$

where $\chi_{n, \pm}^{c}$ are the corresponding positive-energy antifermion eigenstates, which can be derived from the negativeenergy fermion solutions $\chi_{n, \pm}$ via charge conjugation in the usual way, and $\delta \mathcal{H}^{c}$ is the perturbative Hamiltonian for the antifermion, which can also be obtained from $\delta \mathcal{H}$ in a similar way. Applying Eq. (10), the expression for the perturbative energy shifts of the corresponding antifermion is found to have the same form as that of a fermion, except that the spin is reversed, and the contributions are controlled by a set of starred tilde quantities,

$$
\begin{aligned}
\delta E_{n, \pm 1}^{\bar{w}} & \\
= & -\tilde{a}_{w}^{* 0} \pm \sigma \tilde{b}_{w}^{* 3}-\tilde{m}_{F, w}^{* 3} B \mp \sigma \tilde{b}_{F, w}^{* 3} B \\
& \left.+\left(\mp \sigma \tilde{b}_{w}^{\prime * 3}-m_{w} \tilde{c}_{w}^{00}+\left(\tilde{c}_{w}^{11}+\tilde{c}_{w}^{22}\right)_{s}\right]\right) \frac{(2 n+1 \mp \sigma)|q B|}{2 m_{w}^{2}} \\
& +\left( \pm \sigma\left(\tilde{b}_{w}^{* 311}+\tilde{b}_{w}^{* 322}\right)-\frac{1}{m_{w}}\left(\tilde{c}_{w}^{11}+\tilde{c}_{w}^{22}\right)_{s}\right) \frac{(2 n+1)|q B|}{2},
\end{aligned}
$$

where the starred tilde quantities are defined by

$$
\begin{aligned}
\tilde{a}_{w}^{* 0}= & a_{w}^{0}+m_{w} c_{w}^{00}-m_{w} e_{w}^{0}-m_{w}^{2} m_{w}^{(5) 00} \\
& +m_{w}^{2} a_{w}^{(5) 000}+m_{w}^{3} c_{w}^{(6) 0000}-m_{w}^{3} e_{w}^{(6) 000} \\
\tilde{b}_{w}^{* 3}= & b_{w}^{3}-H_{w}^{12}+m_{w} d_{w}^{30}-m_{w} g_{w}^{120}+m_{w}^{2} b_{w}^{(5) 300} \\
& -m_{w}^{2} H_{w}^{(5) 1200}+m_{w}^{3} d_{w}^{(6) 3000}-m_{w}^{3} g_{w}^{(6) 12000} \\
\tilde{m}_{F, w}^{* 3}= & m_{F, w}^{(5) 12}-a_{F, w}^{(5) 012}-m_{w} c_{F, w}^{(6) 0012}+m_{w} e_{F, w}^{(6) 012} \\
\tilde{b}_{F, w}^{* 33}= & b_{F, w}^{(5) 312}-H_{F, w}^{(5) 1212}+m_{w} d_{F, w}^{(6) 3012}-m_{w} g_{F, w}^{(6) 12012} \\
\tilde{b}_{w}^{\prime 3}= & b_{w}^{3}+m_{w}\left(g_{w}^{120}-g_{w}^{012}+g_{w}^{021}\right)-m_{w}^{2} b_{w}^{(5) 300} \\
& +2 m_{w}^{2}\left(H_{w}^{(5) 1200}-H_{w}^{(5) 0102}+H_{w}^{(5) 0201}\right) \\
& -2 m_{w}^{3} d_{w}^{(6) 3000}+3 m_{w}^{3}\left(g_{w}^{(6) 12000}-g_{w}^{(6) 01002}+g_{w}^{(6) 02001}\right), \\
\tilde{c}_{w}^{* 00}= & c_{w}^{00}-m_{w} m_{w}^{(5) 00}+2 m_{w} a_{w}^{(5) 000} \\
& +3 m_{w}^{2} c_{w}^{(6) 0000}-2 m_{w}^{2} e_{w}^{(6) 000}
\end{aligned}
$$

and the corresponding " $11+22$ " types of starred tilde coefficients are given by

$$
\begin{aligned}
\left(\tilde{c}_{w}^{* j j}\right)_{s}= & c_{w}^{j j}+2 m_{w} a_{w}^{(5) j 0 j}+3 m_{w}^{2} c_{w}^{(6) j 00 j}, \\
\left(\tilde{c}_{w}^{* j j}\right)_{s}= & m_{w} a_{w}^{(5) 0 j j}-m_{w} m_{w}^{(5) j j} \\
& +3 m_{w}^{2} c_{w}^{(6) 00 j j}-3 m_{w}^{2} e_{w}^{(6) 0 j j}, \\
\tilde{b}_{w}^{* 3 j j}= & b_{w}^{(5) 3 j j}-H_{w}^{(5) 12 j j} \\
& +3 m_{w} d_{w}^{(6) 30 j j}-3 m_{w} g_{w}^{(6) 120 j j} .
\end{aligned}
$$

In the result (11), the charge sign $\sigma$ of the antifermion is understood to change. Comparing the result (11) to (7), together with the relevant definitions (8), (9), (12), and (13), $\delta E_{n, \pm 1}^{\bar{w}}$ can also be obtained from $\delta E_{n, \pm 1}^{w}$ by reversing the charge sign, the spin orientation, and the signs of all $C P T$-odd coefficients.

We remark in passing that the indices of the tilde coefficients appearing in results (7) and (11) and are defined in (8), (9), (12), and (13) correctly represent their rotation properties. For example, the index pair " 12 " on the right sides of these definitions is antisymmetric [32], which means it transforms like a single " 3 " index under spatial rotations. Coefficients with index " 0 " or index pair " 00 " are invariant under spatial rotations. The dependence of results (7) and (11) on only the index " 0 ", " 3 ", and " $11+22$ " correctly reflects the cylindrical symmetry of the Penning trap.

\section{B. Cyclotron frequency shifts}

One of the key frequencies in a Penning-trap experiment is the cyclotron frequency, which is related to the charge-tomass ratio of the confined particle or antiparticle. The cyclotron frequency is defined as the energy difference between the $n=1$ and $n=0$ Landau levels. For example, for particles $w=e^{-}$and $p$, the cyclotron frequencies in natural units are defined as

$$
\omega_{c}^{e^{-}} \equiv E_{1,-1}^{e^{-}}-E_{0,-1}^{e^{-}}, \quad \omega_{c}^{p} \equiv E_{1,+1}^{p}-E_{0,+1}^{p},
$$

respectively. For the corresponding antiparticles $\bar{w}=e^{+}$ and $\bar{p}$, the cyclotron frequencies are defined in a similar way, except the spin directions are reversed,

$$
\omega_{c}^{e^{+}} \equiv E_{1,+1}^{e^{+}}-E_{0,+1}^{e^{+}}, \quad \omega_{c}^{\bar{p}} \equiv E_{1,-1}^{\bar{p}}-E_{0,-1}^{\bar{p}} .
$$

In the presence of Lorentz and $C P T$ violation, the perturbative energy shifts (7) and (11) can lead to corrections to these cyclotron frequencies. Using the definitions (14) together with the result (7), we find the shifts in the cyclotron frequencies for electrons $w=e^{-}$and protons $w=p$ have the same expression,

$\frac{\delta \omega_{c}^{w}}{e B}=\frac{1}{m_{w}^{2}} \tilde{b}_{w}^{\prime 3}-\frac{1}{m_{w}}\left(\tilde{c}_{w}^{00}+\tilde{c}_{w}^{11}+\tilde{c}_{w}^{22}\right)-\left(\tilde{b}_{w}^{311}+\tilde{b}_{w}^{322}\right)$,

where the tilde coefficients $\tilde{c}_{w}^{j j}$ with $j=1$ or 2 are the sum of the two pieces defined in (9),

$$
\begin{aligned}
\tilde{c}_{w}^{j j}= & \left(\tilde{c}_{w}^{j j}\right)_{s}+\left(\tilde{c}_{w}^{j j}\right)_{s} \\
= & c_{w}^{j j}-2 m_{w} a_{w}^{(5) j 0 j}+3 m_{w}^{2} c_{w}^{(6) j 00 j}-m_{w} a_{w}^{(5) 0 j j} \\
& -m_{w} m_{w}^{(5) j j}+3 m_{w}^{2} c_{w}^{(6) 00 j j}+3 m_{w}^{2} e_{w}^{(6) 0 j j} .
\end{aligned}
$$

The above result (16) shows that the shifts in the cyclotron frequencies due to Lorentz and $C P T$ violation 
depend only on three tilde quantities $\tilde{b}_{w}^{\prime 3}, \tilde{c}_{w}^{00}+\tilde{c}_{w}^{11}+\tilde{c}_{w}^{22}$, and $\tilde{b}_{w}^{311}+\tilde{b}_{w}^{322}$ in the apparatus frame, among which the piece $\tilde{c}_{w}^{00}$ is invariant under rotations but breaks Lorentz symmetry under boosts, while the others violate Lorentz symmetry under both rotations and boosts. All of tilde quantities involve a mixture of $C P T$-even and $C P T$-odd coefficients. No $F$-type coefficients for Lorentz violation appear in result (16) as they produce energy shift contributions that are independent of the Landau level number $n$, as evident from the result (7), and hence are unobservable in the cyclotron frequency shifts.

The corresponding cyclotron frequency shifts for antiparticles $\bar{w}=e^{+}$and $\bar{p}$ can be obtained from (16) by replacing the usual tilde quantities by the corresponding starred ones, given by

$$
\begin{aligned}
\frac{\delta \omega_{c}^{\bar{w}}}{e B}= & -\frac{1}{m_{w}^{2}} \tilde{b}_{w}^{\prime * 3}-\frac{1}{m_{w}}\left(\tilde{c}_{w}^{* 00}+\tilde{c}_{w}^{* 11}+\tilde{c}_{w}^{* 22}\right) \\
& +\left(\tilde{b}_{w}^{* 311}+\tilde{b}_{w}^{* 322}\right),
\end{aligned}
$$

where the starred tilde coefficients $\tilde{c}_{w}^{* j j}$ with $j=1$ or 2 are defined by

$$
\begin{aligned}
\tilde{c}_{w}^{* j j}= & \left(\tilde{c}_{w}^{* j j}\right)_{s}+\left(\tilde{c}_{w}^{* j j}\right)_{s} \\
= & c_{w}^{j j}+2 m_{w} a_{w}^{(5) j 0 j}+3 m_{w}^{2} c_{w}^{(6) j 00 j}+m_{w} a_{w}^{(5) 0 j j} \\
& -m_{w} m_{w}^{(5) j j}+3 m_{w}^{2} c_{w}^{(6) 00 j j}-3 m_{w}^{2} e_{w}^{(6) 0 j j} .
\end{aligned}
$$

It is observed that the only difference between the particle result (16) and the antiparticle result (18) is the sign of all the $C P T$-odd coefficients, as expected.

\section{Transformation under rotations}

The cyclotron frequency shifts (16) and (18) are expressed in the apparatus frame with the positive $\hat{x}_{3}$ axis chosen to be aligned with the magnetic field in the trap. However, this frame is noninertial due to the Earth's rotation. To compare results from different experiments searching for Lorentz and CPT violation, a standard canonical frame is adopted in the literature which is called the Sun-centered frame [33,34]. In this frame the Cartesian coordinates are labeled by $X^{J} \equiv(X, Y, Z)$, with the $Z$ axis aligned along the Earth's rotation axis, the $X$ axis pointing from the Earth to the Sun, and the time $T$ chosen to have origin at the vernal equinox 2000. The coefficients for Lorentz violation in this frame can be assumed to be constants in time and space [2,9].

To relate the coefficients for Lorentz violation from the Sun-centered frame to the apparatus frame, we introduce a third frame called the standard laboratory frame $x^{j} \equiv(x, y, z)$, in which the $z$ axis points to the local zenith, the $x$ axis is aligned with the local south, and the $y$ axis completes a right-handed coordinate system. The convenient choice of the positive $\hat{x}_{3}$ axis of the apparatus frame as the direction of the magnetic field of the trap may result in a nonzero angle to the $\hat{z}$ axis of the standard laboratory frame. Therefore, to relate the coordinates from the apparatus frame to the Sun-centered frame, we define two rotation matrices $R^{a j}$ and $R^{j J}$, with $R^{a j}$ relating the standard laboratory frame $x^{j} \equiv(x, y, z)$ to the apparatus frame $x^{a} \equiv$ $\left(x^{1}, x^{2}, x^{3}\right)$ by $x^{a}=R^{a j} x^{j}$, and $R^{j J}$ connecting $X^{J} \equiv$ $(X, Y, Z)$ of the Sun-centered frame to $(x, y, z)$ of the standard laboratory frame by $x^{j}=R^{j J} X^{J}$. The expression of $R^{j J}$ is given by $[33,34]$

$R^{j J}=\left(\begin{array}{ccc}\cos \chi \cos \omega_{\oplus} T_{\oplus} & \cos \chi \sin \omega_{\oplus} T_{\oplus} & -\sin \chi \\ -\sin \omega_{\oplus} T_{\oplus} & \cos \omega_{\oplus} T_{\oplus} & 0 \\ \sin \chi \cos \omega_{\oplus} T_{\oplus} & \sin \chi \sin \omega_{\oplus} T_{\oplus} & \cos \chi\end{array}\right)$,

where $\omega_{\oplus} \simeq 2 \pi /(23 \mathrm{~h} 56 \mathrm{~min})$ denotes the sidereal frequency of the Earth's rotation, $T_{\oplus}$ specifies the local sidereal time, and $\chi$ is the colatitude of the laboratory. The rotation $R^{a j}$ can be specified in general by a suitable set of Euler angles $(\alpha, \beta, \gamma)$. Adopting the convenient " $y$-convention" of the rotation [35], $R^{a j}$ is found to have the form,

$$
\begin{aligned}
R^{a j}= & \left(\begin{array}{ccc}
\cos \gamma & \sin \gamma & 0 \\
-\sin \gamma & \cos \gamma & 0 \\
0 & 0 & 1
\end{array}\right) \times\left(\begin{array}{ccc}
\cos \beta & 0 & -\sin \beta \\
0 & 1 & 0 \\
\sin \beta & 0 & \cos \beta
\end{array}\right) \\
& \times\left(\begin{array}{ccc}
\cos \alpha & \sin \alpha & 0 \\
-\sin \alpha & \cos \alpha & 0 \\
0 & 0 & 1
\end{array}\right)
\end{aligned}
$$

Putting the above discussion together, the relation between the coordinates of the apparatus frame and these of the Sun-centered frame can be obtained by the following expression:

$$
x^{a}=R^{a j} x^{j}=R^{a j} R^{j J} x^{J},
$$

which can be used to relate the coefficients for Lorentz violation in these two frames. In the special case where the magnetic field is vertical upward, which means axes $\hat{x}_{3}$ and $\hat{z}$ are in the same direction, the Euler angles become $(\alpha, \beta, \gamma)=(0,0,0)$ and $R^{a j}$ reduces to the identity matrix.

The rotation matrix (20) reveals the dependence on the sidereal time and laboratory geometric location of the coefficients for Lorentz violation observed in the apparatus frame. As a result, experimental observables for Lorentz violation can oscillate at harmonics of the sidereal frequency $\omega_{\oplus}$ of the Earth's rotation and have different expressions for laboratories at different colatitudes. To explicitly illustrate this, we consider a Penning-trap experiment located at colatitude $\chi$ with a magnetic field along the $\hat{z}$ axis. The tilde coefficients for Lorentz violation in the cyclotron frequency shifts (16) and (18) that break Lorentz 
symmetry under rotations are $\tilde{b}_{w}^{\prime 3}, \tilde{c}_{w}^{11}+\tilde{c}_{w}^{22}, \tilde{b}_{w}^{311}+\tilde{b}_{w}^{322}$, $\tilde{b}_{w}^{\prime * 3}, \tilde{c}_{w}^{* 11}+\tilde{c}_{w}^{* 22}$, and $\tilde{b}_{w}^{* 311}+\tilde{b}_{w}^{* 322}$ in the apparatus frame. To express the sidereal-time and geometric dependence, we take tilde quantity $\tilde{c}_{w}^{11}+\tilde{c}_{w}^{22}$ as an example. Applying the rotation (22) with $R^{a j}$ being the identity matrix yields

$$
\begin{aligned}
\tilde{c}_{w}^{11}+\tilde{c}_{w}^{22}= & \cos 2 \omega_{\oplus} T_{\oplus}\left(-\frac{1}{2}\left(\tilde{c}_{w}^{X X}-\tilde{c}_{w}^{Y Y}\right) \sin ^{2} \chi\right) \\
& +\sin 2 \omega_{\oplus} T_{\oplus}\left(-\tilde{c}_{w}^{(X Y)} \sin ^{2} \chi\right) \\
& +\cos \omega_{\oplus} T_{\oplus}\left(-\tilde{c}_{w}^{(X Z)} \sin 2 \chi\right) \\
& +\sin \omega_{\oplus} T_{\oplus}\left(-\tilde{c}_{w}^{(Y Z)} \sin 2 \chi\right) \\
& +\frac{1}{4}\left(\tilde{c}_{w}^{X X}+\tilde{c}_{w}^{Y Y}\right)(3+\cos 2 \chi)+\tilde{c}_{w}^{Z Z} \sin ^{2} \chi,
\end{aligned}
$$

where parentheses on two indices of the coefficients imply symmetrization with a factor of $1 / 2$. For instance, $\tilde{c}_{w}^{(X Y)}=\left(\tilde{c}_{w}^{X Y}+\tilde{c}_{w}^{Y X}\right) / 2$. Result (23) shows that the tilde quantity $\tilde{c}_{w}^{11}+\tilde{c}_{w}^{22}$ can be expressed in terms of the six independent quantities $\tilde{c}_{w}^{(J K)}$ with $J, K=X, Y, Z$ in the Sun-centered frame, producing up to second harmonics in the sidereal frequency of the Earth's rotation. The colatitude dependence is shown by the factors appearing in the amplitudes of the harmonic oscillations.

If magnetic fields in different directions are used in a Penning trap, the tilde quantities $\tilde{b}_{w}^{\prime 3}, \tilde{c}_{w}^{11}+\tilde{c}_{w}^{22}, \tilde{b}_{w}^{311}+\tilde{b}_{w}^{322}$, $\tilde{b}_{w}^{\prime * 3}, \tilde{c}_{w}^{* 11}+\tilde{c}_{w}^{* 22}$, and $\tilde{b}_{w}^{* 311}+\tilde{b}_{w}^{* 322}$ in the apparatus frame can have different transformations into the Sun-centered frame. In a typical Penning-trap experiment, the magnetic field is oriented either horizontally or vertically. For analysis reference, we present in Appendix $\mathrm{C}$ the explicit transformation results of these tilde quantities for the above two field orientations. These transformation results show that for a given fermion of species $w$ there are a total of 27 independent tilde coefficients $\tilde{b}_{w}^{\prime J}, \tilde{c}_{w}^{(J K)}$, and $\tilde{b}_{w}^{J(K L)}$ in the Sun-centered frame that are related to the cyclotron frequency shifts in a Penning-trap experiment. Taking into account the corresponding antifermion $\bar{w}$, an additional 27 independent components can be accessed via $\tilde{b}_{w}^{\prime * J}, \tilde{c}_{w}^{*(J K)}$, and $\tilde{b}_{w}^{* J(K L)}$

A different type of time variation of the coefficients for Lorentz violation can arise from the revolution of the Earth about the Sun. This includes the effects from the boost $\beta_{\oplus} \approx 10^{-4}$ of the Earth relative to the Sun, and the boost $\beta_{L} \approx 10^{-6}$ of the laboratory due to the Earth's rotation. As studied in the literature [24,25,36-38], these effects are suppressed by one or more powers of these boost factors compared to these from rotations and hence can be treated as negligible in the present work.

\section{Experimental signals}

The SME can produce various Lorentz- and $C P T$ violating effects in Penning-trap experiments involving confined particles or antiparticles. One type of observable signal arises from the time variation of the experimental quantity measured in the laboratory frame. This is because the magnetic field used in the trap sets up a set of instantaneous coordinates of the laboratory frame, which rotates due to the Earth's rotation and hence produces sidereal variations of the measured signals, as discussed above in Sec. III C. Performing a sidereal-variation analysis of the experimental data would permit the study of the constant coefficients in the Sun-centered frame that are related to different harmonic terms in the transformation. For example, from results (16) and (18), together with the related transformations presented in Appendix C, a sidereal-variation analysis of the cyclotron frequency shifts $\delta \omega_{c}^{w}$ and $\delta \omega_{c}^{\bar{w}}$ of the confined particles and antiparticles would offer sensitivities to components of the tilde coefficients $\tilde{b}_{w}^{\prime J}, \tilde{b}_{w}^{\prime * J}, \tilde{c}_{w}^{(J K)}, \tilde{c}_{w}^{*(J K)}, \tilde{b}_{w}^{J(K L)}$ and $\tilde{b}_{w}^{* J(K L)}$.

Another kind of Lorentz- and $C P T$-violating effect appears in comparative measurements between particles and antiparticles, as the frequency shifts due to Lorentz and $C P T$ violation could differ between particles and antiparticles. For example, according to results (16) and (18), the cyclotron frequencies for a particle and its antiparticle are shifted differently, with the contributions controlled by two different sets of tilde quantities. In a Penning trap experiment using a same magnetic field, the only difference between $\delta \omega_{c}^{w}$ and $\delta \omega_{c}^{\bar{w}}$ is the sign for the $C P T$-odd coefficients, so a comparison between these two frequency shifts $\Delta \omega_{c}^{w}=\delta \omega_{c}^{w}-\delta \omega_{c}^{\bar{w}}$ would permit the cancellations of all the $C P T$-even effects, making it a clean test of $C P T$ symmetry. Together with the sidereal-variation analysis discussed above, different components of these $C P T$-odd tilde coefficients in results (16) and (18) can be extracted in principle.

In this work, we discuss the above two types of signals in Penning-trap experiments involving measurements of the charge-to-mass ratios of a particle and an antiparticle. In a Lorentz-invariant scenario, conventional quantum electrodynamics predicts that the charge-to-mass ratio of a particle or an antiparticle is related to its cyclotron frequency by

$$
\frac{|q|}{m}=\frac{\omega_{c}}{B} .
$$

By measuring the cyclotron frequency of a particle or an antiparticle in a known magnetic field, its charge-to-mass ratio is then determined. Note that in Lorentz-invariant quantum field theory, the definitions of the charge and mass for a particle or antiparticle are based on the coupling constants characterizing the related interaction strength. Therefore, the charge-to-mass ratio is an intrinsic property of a particle or antiparticle which does not vary by the local experimental conditions, such as the field configuration in the trap or the location of the laboratory. Note also that both the charge and mass are Lorentz scalars in quantum field 
theory and are invariant under Lorentz transformation. It follows that the charge, mass and the resulting chargeto-mass ratio defined in the context of Lorentz-invariant quantum field theory are unchanged even though Lorentz symmetry is broken.

For comparative measurements, suppose a Penning-trap experiment uses a same magnetic field to measure the cyclotron frequencies of a particle $w$ and its corresponding antiparticle $\bar{w}$ simultaneously. The result can then be related to the comparison of the charge-to-mass ratios between a particle and its antiparticle,

$$
\frac{(|q| / m)_{\bar{w}}}{(|q| / m)_{w}}-1=\frac{\omega_{c}^{\bar{w}}}{\omega_{c}^{w}}-1 .
$$

In the Lorentz- and $C P T$-invariant scenario, this difference is identically zero by the $C P T$ theorem. Therefore, in the context of Lorentz-invariant quantum field theory (which also implies $C P T$ invariance), the experimental interpreted quantity $(|q| / m)_{\bar{w}} /(|q| / m)_{w}-1$ is a clean measure of a CPT test.

However, in the presence of Lorentz violation, the cyclotron frequency of a particle or antiparticle is shifted by (16) or (18), respectively. This implies that the measured cyclotron frequency becomes an experiment-dependent quantity, as a function of the local sidereal time, the colatitude of the laboratory, and both the direction and magnitude of the magnetic field used in the trap. As a result, the difference (25) does not vanish in general and become an experiment-dependent quantity, given by

$$
\frac{(|q| / m)_{\bar{w}}}{(|q| / m)_{w}}-1 \leftrightarrow \frac{\omega_{c}^{\bar{w}}}{\omega_{c}^{w}}-1=\frac{\delta \omega_{c}^{\bar{w}}-\delta \omega_{c}^{w}}{\omega_{c}^{w}},
$$

where $\leftrightarrow$ means the charge-to-mass ratio comparison reported by the experiments are obtained by interpreting the measured difference $\omega_{c}^{\bar{w}} / \omega_{c}^{w}-1$, as the relation (24) becomes an approximation in the presence of Lorentz violation. On the right side of Eq. (26), the Lorentz- and $C P T$-invariant pieces in the measured cyclotron frequencies are exactly canceled by the $C P T$ theorem if a same magnetic field is used. From the cyclotron frequency shifts (16) and (18), together with the transformation results in Appendix C, the difference $\delta \omega_{c}^{w}-\delta \omega_{c}^{w}$ on the right side of the equation (26) contains only $C P T$-odd coefficients for Lorentz violation, producing pure $C P T$-violating effects. In a general case where a comparison is made by using different magnetic fields, Eq. (26) becomes

$\frac{(|q| / m)_{\bar{w}}}{(|q| / m)_{w}}-1 \leftrightarrow \frac{\omega_{c}^{\bar{w}} / B^{*}}{\omega_{c}^{w} / B}-1=\frac{\delta \omega_{c}^{\bar{w}} / B^{*}-\delta \omega_{c}^{w} / B}{\omega_{c}^{w} / B}$,

where $B^{*}$ and $B$ are the strengths of the magnetic fields used for measuring $\omega_{c}^{\bar{w}}$ and $\omega_{c}^{w}$, respectively. It is clear that the $C P T$-even coefficients on the right side of Eq. (27) do not exactly cancel out due to the different magnetic strengths, even when the magnetic fields are in the same direction. Therefore, in this case the experimental interpreted quantity $(|q| / m)_{\bar{w}} /(|q| / m)_{w}-1$ is not a clean measure of a $C P T$ test.

Now we conclude that whether the experimental interpreted quantity $(|q| / m)_{\bar{w}} /(|q| / m)_{w}-1$ is a clean test of $C P T$ symmetry depends on the context of the relevant theory. In a Lorentz-invariant quantum field theory, it can be used as a clean test of $C P T$ symmetry. However, in a general Lorentz-violating scenario, it is a clean measure of a $C P T$ test only if a same magnetic field is used. Similar discussions for the $g$ factor comparisons have also been addressed in Sec. III B 1 in Ref. [8]. The key point is that the experimental quantity $(|q| / m)_{\bar{w}} /(|q| / m)_{w}-1$ is obtained by interpreting the measured difference $\omega_{c}^{\bar{w}} / \omega_{c}^{w}-1$ in the context of conventional quantum electrodynamics. Lorentz violation does not modify the theoretical value of the charge-to-mass ratio for a particle or antiparticle, which is defined via conventional quantum electrodynamics. What it affects are the measured cyclotron frequencies that are used by experiments to interpret the charge-to-mass ratios and their comparison between a particle and an antiparticle.

At the end of this subsection, we discuss a subtlety arising from Penning-trap experiments comparing the charge-to-mass ratios between an antiproton and a proton. As the two particles have opposite charges, the measurements of their cyclotron frequencies using the same trap requires the reversal of the quadruple electric field. To facilitate the experiment by eliminating the systematic shifts caused by polarity switching of the trapping voltages, a hydrogen ion $\left(\mathrm{H}^{-}\right)$is used as a proxy for the proton. This allows a relatively fast exchange between hydrogen ions and antiprotons. The comparison of the charge-to-mass ratios between an antiproton and a proton can be related to that between an antiproton and a hydrogen ion by

$\frac{(|q| / m)_{\bar{p}}}{(|q| / m)_{p}}-1=\frac{(|q| / m)_{\bar{p}}}{R(|q| / m)_{\mathrm{H}^{-}}}-1 \leftrightarrow \frac{\delta \omega_{c}^{\bar{p}}-R \delta \omega_{c}^{\mathrm{H}^{-}}}{R \omega_{c}^{\mathrm{H}^{-}}}$,

where $R=m_{\mathrm{H}^{-}} / m_{p}=1.001089218754$ is the mass ratio of a hydrogen ion and a proton [5], $\omega_{c}^{\mathrm{H}^{-}}$and $\delta \omega_{c}^{\mathrm{H}^{-}}$are the cyclotron frequency and the corresponding shifts for the hydrogen ion, respectively.

The cyclotron frequency shift $\delta \omega_{c}^{\mathrm{H}^{-}}$for a hydrogen ion in the above result can be obtained by taking $w=\mathrm{H}^{-}$in the expression (16). The related coefficients for Lorentz violation become the effective ones for a hydrogen ion. In the framework of the SME, effective coefficients for a composite particle can be expressed in terms of the corresponding fundamental coefficients for Lorentz violation for its constituents. In our case, the related fundamental coefficients are these for electrons and protons. Deriving the exact relations between these coefficients can be challenging 
due to nonperturbative issues involving binding effects for the composite particle. However, a good approximation to these relations can be found by taking the lowest-order perturbation theory and treating the hydrogen ion wave function as a product of the wave functions of a proton and two electrons. Ignoring the binding energies, the energy shifts of the hydrogen ion due to Lorentz and $C P T$ violation can be approximated as the sum of these for its constituents. The corresponding approximated relation of the shifts in their cyclotron frequencies can be found as

$$
\delta \omega_{c}^{\mathrm{H}^{-}} \approx \delta \omega_{c}^{p}+2 \delta \omega_{c}^{e^{-}} .
$$

Substituting relation (29) into the result (28) yields

$$
\frac{(|q| / m)_{\bar{p}}}{(|q| / m)_{p}}-1 \approx \frac{\delta \omega_{c}^{\bar{p}}-R \delta \omega_{c}^{p}-2 R \delta \omega_{c}^{e^{-}}}{R \omega_{c}^{\mathrm{H}^{-}}}
$$

The above result shows that Penning-trap experiments comparing the charge-to-mass ratios between an antiproton and a proton by using a hydrogen ion as a proxy for the proton are sensitive not only to the SME coefficients for protons, but also provide access to these for electrons. From the transformation results presented in Appendix C, the related coefficients for Lorentz violation in the Suncentered frame are these 81 independent tilde quantities $\tilde{b}_{p}^{\prime J}, \tilde{c}_{p}^{(J K)}, \tilde{b}_{p}^{J(K L)}, \tilde{b}_{p}^{\prime * J}, \tilde{c}_{p}^{*(J K)}, \tilde{b}_{p}^{* J(K L)}, \tilde{b}_{e}^{\prime J}, \tilde{c}_{e}^{(J K)}$, and $\tilde{b}_{e}^{J(K L)}$. Using the expression (30) the published results from experiments comparing the charge-to-mass ratios between an antiproton and a hydrogen ion can be adopted to set bounds on the relevant coefficients for Lorentz violation.

\section{E. Experimental sensitivities}

In this subsection, we focus on the analysis of two Penning trap experiments comparing the charge-to-mass ratios between an antiproton and a proton and provide the explicit combinations of the tilde coefficients that are sensitive to each individual experiment. Taking the published results we constrain the relevant tilde coefficients in the Sun-centered frame.

\section{The ATRAP experiment}

In the ATRAP experiment located at CERN, Gabrielse and his collaboration compared the charge-to-mass ratios between an antiproton and a proton to a precision of $90 \mathrm{ppt}$ using a simultaneously trapped antiproton and hydrogen ion in a vertical uniform magnetic field $B=5.85 \mathrm{~T}$ [12]. The reported precision was obtained by analyzing the measurements of the cyclotron frequencies in a timeaveraged way, so any effects in the difference (30) that are dependent of sidereal time averaged out. This implies that the published precision can be used to constrain only the tilde coefficients that appear in the constant terms in the transformation results listed in Appendix C. In principle, a sidereal-variation analysis of the experiment data could also be performed to obtain the constraints on other components of the tilde coefficients that are related to the harmonic terms in the transformation results. For the reference of future sidereal-variation analysis of the ATRAP experiment, we take $\chi=43.8^{\circ}$ for the laboratory colatitude and present in Table I the explicit combinations of the tilde coefficients for all the related harmonics in the transformation results of the tilde quantities $\tilde{b}_{w}^{\prime 3}, \tilde{c}_{w}^{11}+\tilde{c}_{w}^{22}$, and $\tilde{b}_{w}^{311}+\tilde{b}_{w}^{322}$. The table is organized as follows. The first column specifies the name of the experiment, and the second column gives the relevant tilde quantities in the laboratory frame. The corresponding combinations of the tilde coefficients in the Sun-centered frame are listed in the third column, with the associated harmonics displaced in the final column.

Using expression (30) together with the reported precision of $90 \mathrm{ppt}$ for $(|q| / m)_{\bar{p}} /(|q| / m)_{p}-1$ and $\omega_{c}^{\mathrm{H}^{-}}=$ $2 \pi \times 89.3 \mathrm{MHz}$ for the ATRAP experiment, we obtain the following limit in natural units:

$\left|\delta \omega_{c}^{\bar{p}}-1.001 \delta \omega_{c}^{p}-2.002 \delta \omega_{c}^{e^{-}}\right|_{\text {const }} \lesssim 3.33 \times 10^{-26} \mathrm{GeV}$,

where the subscript "const" implies this limit is only for the tilde coefficients appearing in the constant terms in the transformations results.

\section{The BASE experiment}

Another Penning-trap experiment at CERN by the BASE Collaboration recently improved the same comparison to the record sensitivity of $69 \mathrm{ppt}$ [5]. Since the BASE experiment also used a hydrogen ion as a proxy of a proton, the expression (30) still holds. The trap used a horizontal magnetic field $B=1.946 \mathrm{~T}$ oriented $60^{\circ}$ east of north. This implies that both matrices (20) and (21) are needed for determining the combination of the tilde coefficients in the Sun-centered frame. The corresponding Euler angles for a horizontal magnetic field with an angle $\theta$ from the local south in the counterclockwise direction are found to be $(\alpha, \beta, \gamma)=(\theta, \pi / 2,0)$. Taking $\theta=2 \pi / 3$ and $\chi=43.8^{\circ}$ for the BASE experiment we also include in Table I the related explicit transformation results for the tilde quantities $\tilde{b}_{w}^{\prime 3}, \tilde{c}_{w}^{11}+\tilde{c}_{w}^{22}$, and $\tilde{b}_{w}^{311}+\tilde{b}_{w}^{322}$.

Different from the ATRAP experiment, the experimental data of the charge-to-mass ratio comparison for the BASE experiment were analyzed to search for both time-averaged effects and sidereal variations in the first harmonic of the Earth's rotation frequency, so the reported sensitivities from the experiment can be taken to set bounds on both the tilde coefficients appearing in the constant terms and these in the first harmonic of the oscillations. Using $69 \mathrm{ppt}$ for the timeaveraged precision and $720 \mathrm{ppt}$ for the limit of the first 
TABLE I. Combinations of tilde coefficients in the Sun-centered frame for both the ATRAP and BASE experiments.

\begin{tabular}{|c|c|c|c|}
\hline Experiment & Laboratory frame & Sun-centered frame & Harmonic \\
\hline ATRAP & $\tilde{c}_{w}^{11}+\tilde{c}_{w}^{22}$ & $\begin{array}{c}0.72 \tilde{b}_{w}^{\prime Z} \\
0.69 \tilde{b}_{w}^{\prime X} \\
0.69 \tilde{b}_{w}^{\prime Y} \\
0.76\left(\tilde{c}_{w}^{X X}+\tilde{c}_{w}^{Y Y}\right)^{\prime Y}+0.48 \tilde{c}_{w}^{Z Z} \\
-1.0 \tilde{c}_{w}^{(X Z)} \\
-1.0 \tilde{c}_{w}^{(Y Z)} \\
-0.24\left(\tilde{c}_{w}^{X X}-\tilde{c}_{w}^{Y Y}\right) \\
-0.48 \tilde{c}_{w}^{(X Y)} \\
-0.35\left(\tilde{b}_{w}^{X(X Z)}+\tilde{b}_{w}^{Y(Y Z)}-\tilde{b}_{w}^{Z Z Z}\right)+0.55\left(\tilde{b}_{w}^{Z X X}+\tilde{b}_{w}^{Z Y Y}\right) \\
0.44 \tilde{b}_{w}^{X X X}+0.61 \tilde{b}_{w}^{X Y Y}+0.33 \tilde{b}_{w}^{X Z Z}-0.17 \tilde{b}_{w}^{Y(X Y)} w-0.72 \tilde{b}_{w}^{Z(X Z)} \\
-0.17 \tilde{b}_{w}^{X(X Y)}+0.61 \tilde{b}_{w}^{Y X X}+0.44 \tilde{b}_{w}^{Y Y Y}+0.33 \tilde{b}_{w}^{Y Z Z}-0.72 \tilde{b}_{w}^{Z(Y Z)} \\
-0.35\left(\tilde{b}_{w}^{X(X Z)}-\tilde{b}_{w}^{Y(Y Z)}\right)-0.17\left(\tilde{b}_{w}^{Z X X}-\tilde{b}_{w}^{Z Y Y}\right) \\
-0.35\left(\tilde{b}_{w}^{X(Y Z)}+\tilde{b}_{w}^{Y(X Z)}+\tilde{b}_{w}^{Z(X Y)}\right) \\
-0.08\left(\tilde{b}_{u}^{X X X}-\tilde{b}_{w}^{X Y Y}\right)+0.17 \tilde{b}_{w}^{Y(X Y)} \\
-0.17 \tilde{b}_{w}^{X(X Y)}-0.08\left(\tilde{b}_{w}^{Y X X}-\tilde{b}_{w}^{Y Y Y}\right)\end{array}$ & $\begin{array}{l}1 \\
\cos \omega_{\oplus} T_{\oplus} \\
\sin \omega_{\oplus} T_{\oplus} \\
1 \\
\cos \omega_{\oplus} T_{\oplus} \\
\sin \omega_{\oplus} T_{\oplus} \\
\cos 2 \omega_{\oplus} T_{\oplus} \\
\sin 2 \omega_{\oplus} T_{\oplus} \\
1 \\
\cos \omega_{\oplus} T_{\oplus} \\
\sin \omega_{\oplus} T_{\oplus} \\
\cos 2 \omega_{\oplus} T_{\oplus} \\
\sin 2 \omega_{\oplus} T_{\oplus} \\
\cos 3 \omega_{\oplus} T_{\oplus} \\
\sin 3 \omega_{\oplus} T_{\oplus}\end{array}$ \\
\hline BASE & $\begin{array}{c}\tilde{b}_{w}^{\prime 3} \\
\tilde{c}_{w}^{11}+\tilde{c}_{w}^{22}\end{array}$ & 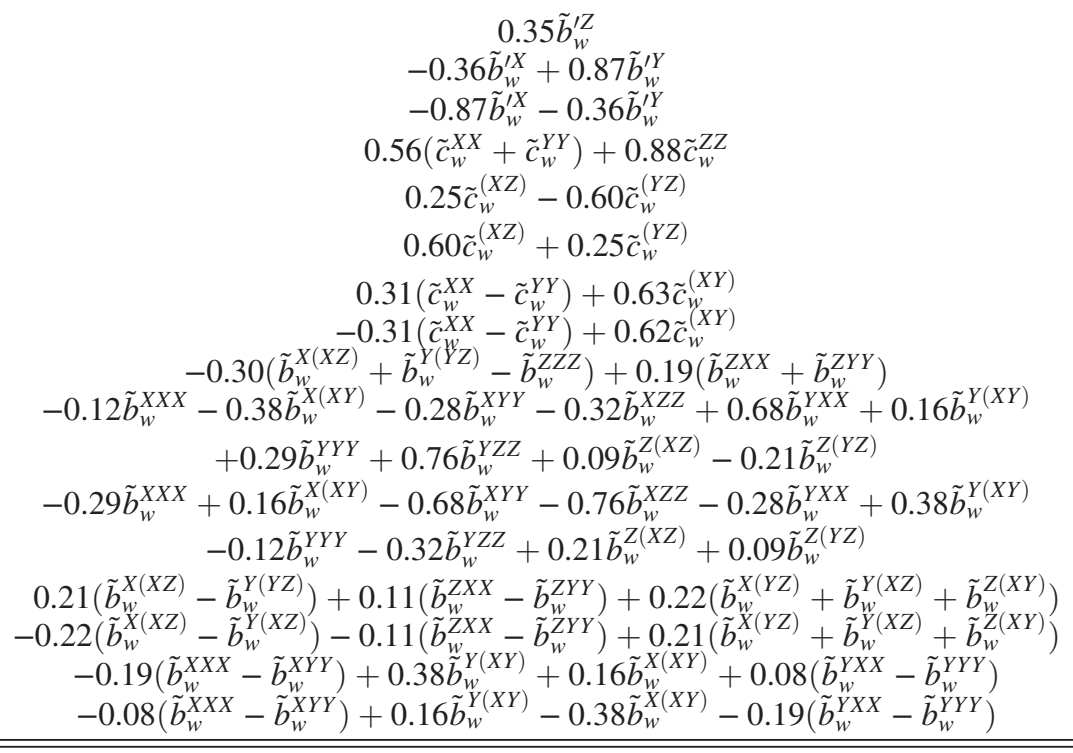 & $\begin{array}{c}1 \\
\cos \omega_{\oplus} T_{\oplus} \\
\sin \omega_{\oplus} T_{\oplus} \\
1 \\
\cos \omega_{\oplus} T_{\oplus} \\
\sin \omega_{\oplus} T_{\oplus} \\
\cos 2 \omega_{\oplus} T_{\oplus} \\
\sin 2 \omega_{\oplus} T_{\oplus} \\
1 \\
\cos \omega_{\oplus} T_{\oplus}\end{array}$ \\
\hline
\end{tabular}

harmonic amplitude, together with $\omega_{c}^{\mathrm{H}^{-}}=2 \pi \times 29.635 \mathrm{MHz}$ from the experiment, expression (30) yields

$\left|\delta \omega_{c}^{\bar{p}}-1.001 \delta \omega_{c}^{p}-2.002 \delta \omega_{c}^{e^{-}}\right|_{\text {const }} \lesssim 8.46 \times 10^{-27} \mathrm{GeV}$

and

$\left|\delta \omega_{c}^{\bar{p}}-1.001 \delta \omega_{c}^{p}-2.002 \delta \omega_{c}^{e^{-}}\right|_{1 \mathrm{st}} \lesssim 8.83 \times 10^{-26} \mathrm{GeV}$

in natural units, where the subscript "const" in the limit (32) has the same meaning as what in (31), while the subscript "1st" in the limit (33) represents the amplitude of the first harmonic in the sidereal variation.

Some intuition about the scope of the constraints on the individual components of the tilde coefficients appearing in limits (31), (32), and (33) can be obtained by assuming that only one individual tilde coefficient to be nonzero at a time and extracting its resulting constraint, which is a common practice adopted in many subfields searching for Lorentz and $C P T$ violation [4]. Using the limit (31) from the ATRAP experiment, a total of 27 independent tilde coefficients for Lorentz violation are constrained. For the BASE experiment, 69 constraints are obtained on the independent tilde coefficients for Lorentz violation from limits (32) and (33). To summarize the results, we list in Table II the individual constraints on the tilde coefficients in the Suncentered frame, with the first column listing the individual components, the second column presenting the corresponding constraint on the modulus of each one, and the third column specifying the related experiment. Note that when a component of the tilde coefficients is constrained by both the ATRAP and the BASE experiment, we only include the more stringent one in Table II. 
TABLE II. Constraints on tilde coefficients for Lorentz violation from the ATRAP and the BASE experiments.

\begin{tabular}{|c|c|c|}
\hline Coefficient & Constraint & Experiment \\
\hline$\left|\tilde{b}_{e}^{\prime Z}\right|$ & $<1.7 \times 10^{-17} \mathrm{GeV}$ & ATRAP \\
\hline$\left|\tilde{c}_{e}^{X X}\right|$ & $<3.2 \times 10^{-14}$ & ATRAP \\
\hline$\left|\tilde{c}_{e}^{Y Y}\right|$ & $<3.2 \times 10^{-14}$ & ATRAP \\
\hline$\left|\tilde{c}_{e}^{Z Z}\right|$ & $<2.1 \times 10^{-14}$ & BASE \\
\hline$\left|\tilde{b}_{e}^{X(X Z)}\right|$ & $<1.2 \times 10^{-10} \mathrm{GeV}^{-1}$ & BASE \\
\hline$\left|\tilde{b}_{e}^{Y(Y Z)}\right|$ & $<1.2 \times 10^{-10} \mathrm{GeV}^{-1}$ & BASE \\
\hline$\left|\tilde{b}_{e}^{Z Z Z}\right|$ & $<1.2 \times 10^{-10} \mathrm{GeV}^{-1}$ & BASE \\
\hline$\left|\tilde{b}_{e}^{Z X X}\right|$ & $<8.8 \times 10^{-11} \mathrm{GeV}^{-1}$ & ATRAP \\
\hline$\left|\tilde{b}_{e}^{Z Y Y}\right|$ & $<8.8 \times 10^{-11} \mathrm{GeV}^{-1}$ & ATRAP \\
\hline$\left|\tilde{b}_{e}^{\prime X}\right|$ & $<1.1 \times 10^{-16} \mathrm{GeV}$ & BASE \\
\hline$\left|\tilde{b}_{e}^{\prime Y}\right|$ & $<1.1 \times 10^{-16} \mathrm{GeV}$ & BASE \\
\hline$\left|\tilde{c}_{e}^{(X Z)}\right|$ & $<3.0 \times 10^{-13}$ & BASE \\
\hline$\left|\tilde{c}_{e}^{(Y Z)}\right|$ & $<3.0 \times 10^{-13}$ & BASE \\
\hline$\left|\tilde{b}_{e}^{X X X}\right|$ & $<1.2 \times 10^{-9} \mathrm{GeV}^{-1}$ & BASE \\
\hline$\left|\tilde{b}_{e}^{X(X Y)}\right|$ & $<9.3 \times 10^{-10} \mathrm{GeV}^{-1}$ & BASE \\
\hline$\left|\tilde{b}_{e}^{X Y Y}\right|$ & $<5.2 \times 10^{-10} \mathrm{GeV}^{-1}$ & BASE \\
\hline$\left|\tilde{b}_{e}^{X Z Z}\right|$ & $<4.6 \times 10^{-10} \mathrm{GeV}^{-1}$ & BASE \\
\hline$\left|\tilde{b}_{e}^{Y X X}\right|$ & $<5.2 \times 10^{-10} \mathrm{GeV}^{-1}$ & BASE \\
\hline$\left|\tilde{b}_{e}^{Y(X Y)}\right|$ & $<9.3 \times 10^{-10} \mathrm{GeV}^{-1}$ & BASE \\
\hline$\left|\tilde{b}_{e}^{Y Y Y}\right|$ & $<1.2 \times 10^{-9} \mathrm{GeV}^{-1}$ & BASE \\
\hline$\left|\tilde{b}_{e}^{Y Z Z}\right|$ & $<4.6 \times 10^{-10} \mathrm{GeV}^{-1}$ & BASE \\
\hline$\left|\tilde{b}_{e}^{Z(X Z)}\right|$ & $<1.7 \times 10^{-9} \mathrm{GeV}^{-1}$ & BASE \\
\hline$\left|\tilde{b}_{e}^{Z(Y Z)}\right|$ & $<1.7 \times 10^{-9} \mathrm{GeV}^{-1}$ & BASE \\
\hline$\left|\tilde{b}_{p}^{\prime Z}\right|,\left|\tilde{b}_{p}^{\prime * Z}\right|$ & $<1.2 \times 10^{-10} \mathrm{GeV}$ & ATRAP \\
\hline$\left|\tilde{c}_{p}^{X X}\right|,\left|\tilde{c}_{p}^{* X X}\right|$ & $<1.2 \times 10^{-10}$ & ATRAP \\
\hline$\left|\tilde{c}_{p}^{Y Y}\right|,\left|\tilde{c}_{p}^{* Y Y}\right|$ & $<1.2 \times 10^{-10}$ & ATRAP \\
\hline$\left|\tilde{c}_{p}^{Z Z}\right|,\left|\tilde{c}_{p}^{* Z Z}\right|$ & $<7.9 \times 10^{-11}$ & BASE \\
\hline$\left|\tilde{b}_{p}^{X(X Z)}\right|,\left|\tilde{b}_{p}^{* X(X Z)}\right|$ & $<2.4 \times 10^{-10} \mathrm{GeV}^{-1}$ & BASE \\
\hline$\left|\tilde{b}_{p}^{Y(Y Z)}\right|,\left|\tilde{b}_{p}^{* Y(Y Z)}\right|$ & $<2.4 \times 10^{-10} \mathrm{GeV}^{-1}$ & BASE \\
\hline$\left|\tilde{b}_{p}^{Z Z Z}\right|,\left|\tilde{b}_{p}^{* Z Z Z}\right|$ & $<2.4 \times 10^{-10} \mathrm{GeV}^{-1}$ & BASE \\
\hline$\left|\tilde{b}_{p}^{Z X X}\right|,\left|\tilde{b}_{p}^{* Z X X}\right|$ & $<1.8 \times 10^{-10} \mathrm{GeV}^{-1}$ & ATRAP \\
\hline$\left|\tilde{b}_{p}^{Z Y Y}\right|,\left|\tilde{b}_{p}^{* Z Y Y}\right|$ & $<1.8 \times 10^{-10} \mathrm{GeV}^{-1}$ & ATRAP \\
\hline$\left|\tilde{b}_{p}^{\prime X}\right|,\left|\tilde{b}_{p}^{\prime * X}\right|$ & $<7.2 \times 10^{-10} \mathrm{GeV}$ & BASE \\
\hline$\left|\tilde{b}_{p}^{\prime Y}\right|,\left|\tilde{b}_{p}^{\prime * Y}\right|$ & $<7.2 \times 10^{-10} \mathrm{GeV}$ & BASE \\
\hline$\left|\tilde{c}_{p}^{(X Z)}\right|,\left|\tilde{c}_{p}^{*(X Z)}\right|$ & $<1.1 \times 10^{-9}$ & BASE \\
\hline$\left|\tilde{c}_{p}^{(Y Z)}\right|,\left|\tilde{c}_{p}^{*(Y Z)}\right|$ & $<1.1 \times 10^{-9}$ & BASE \\
\hline$\left|\tilde{b}_{p}^{X X X}\right|,\left|\tilde{b}_{p}^{* X X X}\right|$ & $<2.4 \times 10^{-9} \mathrm{GeV}^{-1}$ & BASE \\
\hline$\left|\tilde{b}_{p}^{X(X Y)}\right|,\left|\tilde{b}_{p}^{* X(X Y)}\right|$ & $<1.9 \times 10^{-9} \mathrm{GeV}^{-1}$ & BASE \\
\hline$\left|\tilde{b}_{p}^{X Y Y}\right|,\left|\tilde{b}_{p}^{* X Y Y}\right|$ & $<1.1 \times 10^{-9} \mathrm{GeV}^{-1}$ & BASE \\
\hline$\left|\tilde{b}_{p}^{X Z Z}\right|,\left|\tilde{b}_{p}^{* X Z Z}\right|$ & $<9.3 \times 10^{-9} \mathrm{GeV}^{-1}$ & BASE \\
\hline$\left|\tilde{b}_{p}^{Y X X}\right|,\left|\tilde{b}_{p}^{* Y X X}\right|$ & $<1.1 \times 10^{-9} \mathrm{GeV}^{-1}$ & BASE \\
\hline$\left|\tilde{b}_{p}^{Y(X Y)}\right|,\left|\tilde{b}_{p}^{* Y(X Y)}\right|$ & $<1.9 \times 10^{-9} \mathrm{GeV}^{-1}$ & BASE \\
\hline$\left|\tilde{b}_{p}^{Y Y Y}\right|,\left|\tilde{b}_{p}^{* Y Y Y}\right|$ & $<2.4 \times 10^{-9} \mathrm{GeV}^{-1}$ & BASE \\
\hline$\left|\tilde{b}_{p}^{Y Z Z}\right|,\left|\tilde{b}_{p}^{* Y Z Z}\right|$ & $<9.3 \times 10^{-10} \mathrm{GeV}^{-1}$ & BASE \\
\hline$\left|\tilde{b}_{p}^{Z(X Z)}\right|,\left|\tilde{b}_{p}^{* Z(X Z)}\right|$ & $<3.4 \times 10^{-9} \mathrm{GeV}^{-1}$ & BASE \\
\hline$\left|\tilde{b}_{p}^{Z(Y Z)}\right|,\left|\tilde{b}_{p}^{* Z(Y Z)}\right|$ & $<3.4 \times 10^{-9} \mathrm{GeV}^{-1}$ & BASE \\
\hline
\end{tabular}

Table II shows that 69 of the 81 independent components of the tilde coefficients that are related to Penningtrap experiments comparing charge-to-mass ratios of an antiproton and a proton can be bounded using the published results. The other 12 components lie in the second and third harmonics of the transformation results given by Appendix $\mathrm{C}$ and Table I. Therefore, in order to extract all the related constraints on the tilde coefficients, a full sidereal-variation analysis must be performed on the experimental data. The above 69 tilde coefficients listed in Table II are analyzed for the first time as they lie in different coefficient space compared to existing ones that are related to Penning-trap experiments comparing the $g$ factors between particles and antiparticles [8].

\section{SUMMARY}

In this work, we applied the general theory of quantum electrodynamics with Lorentz- and $C P T$-violating operators of mass dimensions up to six to Penning-trap experiments comparing the charge-to-mass ratios between antiprotons and protons. Using perturbation theory, we derived the dominant Lorentz- and $C P T$ violating contributions (7) and (11) to the energy levels of the confined particles and antiparticles, which enabled us to determine the corresponding cyclotron frequency shifts (16) and (18). Relating the experimental interpreted charge-to-mass ratio comparisons to the cyclotron frequency shifts, we addressed the issue of a CPT test and concluded that it depends on the context of the relevant theory. We found in Eq. (30) that the coefficients for Lorentz violation that are sensitive to the charge-to-mass ratio comparison between antiprotons and protons are the 81 independent tilde quantities $\tilde{b}_{p}^{\prime J}, \tilde{c}_{p}^{(J K)}, \tilde{b}_{p}^{J(K L)}, \tilde{b}_{p}^{\prime * J}$, $\tilde{c}_{p}^{*(J K)}, \tilde{b}_{p}^{* J(K L)}, \tilde{b}_{e}^{\prime J}, \tilde{c}_{e}^{(J K)}$, and $\tilde{b}_{e}^{J(K L)}$ in the Sun-centered frame. Using published results from the ATRAP and the BASE experiments, we obtained first-time constraints on 69 of them and summarized the results in Table II. To set bounds to the other 12 components of the tilde coefficients for Lorentz violation, a full sidereal-variation analysis of the experimental data is required. The highprecision measurements and excellent coverage of the SME coefficients offered by current and forthcoming Penning-trap experiments provide strong motivations to continue the searches for possible Lorentz- and CPTviolating signals.

\section{ACKNOWLEDGMENTS}

We thank V. A. Kostelecký for useful discussions and $\mathrm{K}$. Andresen for reading the manuscript. This work was supported in part by the Gettysburg College CrossDisciplinary Science Institute (X-SIG). 


\section{APPENDIX A: FULL LAGRANGE DENSITY FOR $\boldsymbol{d} \leq \mathbf{6}$}

The full Lagrange density (1) with Lorentz-violating operators of mass dimensions $d \leq 6$ can be taken as the sum of the conventional Lorentz-invariant QED Lagrange density $\mathcal{L}_{0}$ and a series of Lorentz-violating terms $\mathcal{L}^{(d)}$ of mass dimension $d, \mathcal{L}_{\psi}=\mathcal{L}_{0}+\mathcal{L}^{(3)}+\mathcal{L}^{(4)}+\mathcal{L}^{(5)}+\mathcal{L}^{(6)}+\ldots$ The explicit results were given in Ref. [8]. Here, we reproduce these terms.

The pieces in the minimal SME Lagrange density $\mathcal{L}^{(3)}$ and $\mathcal{L}^{(4)}$ are

$$
\begin{aligned}
\mathcal{L}^{(3)}=-a^{\mu} \bar{\psi} \gamma_{\mu} \psi-b^{\mu} \bar{\psi} \gamma_{5} \gamma_{\mu} \psi-\frac{1}{2} H^{\mu \nu} \bar{\psi} \sigma_{\mu \nu} \psi \\
\mathcal{L}^{(4)}=\frac{1}{2} c^{\mu \alpha} \bar{\psi} \gamma_{\mu} i D_{\alpha} \psi+\frac{1}{2} d^{\mu \alpha} \bar{\psi} \gamma_{5} \gamma_{\mu} i D_{\alpha} \psi+\frac{1}{2} e^{\alpha} \bar{\psi} i D_{\alpha} \psi \\
+\frac{1}{2} i f^{\alpha} \bar{\psi} \gamma_{5} i D_{\alpha} \psi+\frac{1}{4} g^{\mu \nu \alpha} \bar{\psi} \sigma_{\mu \nu} i D_{\alpha} \psi+\text { H.c. }
\end{aligned}
$$

The dimension-five Lagrange density $\mathcal{L}^{(5)}$ can be classified into two kinds, $\mathcal{L}^{(5)}=\mathcal{L}_{D}^{(5)}+\mathcal{L}_{F}^{(5)}$, with $\mathcal{L}_{D}^{(5)}$ containing symmetrized covariant derivatives $D_{\alpha}$ and $\mathcal{L}_{F}^{(5)}$ involving the antisymmetric electromagnetic field strength $F_{\alpha \beta}$, given by

$$
\begin{aligned}
\mathcal{L}_{D}^{(5)}= & -\frac{1}{2} m^{(5) \alpha \beta} \bar{\psi} i D_{(\alpha} i D_{\beta)} \psi-\frac{1}{2} i m_{5}^{(5) \alpha \beta} \bar{\psi} \gamma_{5} i D_{(\alpha} i D_{\beta)} \psi \\
& -\frac{1}{2} a^{(5) \mu \alpha \beta} \bar{\psi} \gamma_{\mu} i D_{(\alpha} i D_{\beta)} \psi-\frac{1}{2} b^{(5) \mu \alpha \beta} \bar{\psi} \gamma_{5} \gamma_{\mu} i D_{(\alpha} i D_{\beta)} \psi \\
& -\frac{1}{4} H^{(5) \mu \nu \alpha \beta} \bar{\psi} \sigma_{\mu \nu} i D_{(\alpha} i D_{\beta)} \psi+\text { H.c. },
\end{aligned}
$$

$$
\begin{aligned}
\mathcal{L}_{F}^{(5)}= & -\frac{1}{2} m_{F}^{(5) \alpha \beta} F_{\alpha \beta} \bar{\psi} \psi-\frac{1}{2} i m_{5 F}^{(5) \alpha \beta} F_{\alpha \beta} \bar{\psi} \gamma_{5} \psi \\
& -\frac{1}{2} a_{F}^{(5) \mu \alpha \beta} F_{\alpha \beta} \bar{\psi} \gamma_{\mu} \psi-\frac{1}{2} b_{f}^{(5) \mu \alpha \beta} F_{\alpha \beta} \bar{\psi} \gamma_{5} \gamma_{\mu} \psi \\
& -\frac{1}{4} H_{F}^{(5) \mu \nu \alpha \beta} F_{\alpha \beta} \bar{\psi} \sigma_{\mu \nu} \psi .
\end{aligned}
$$

For $d=6$, there are three types of terms, $\mathcal{L}^{(6)}=$ $\mathcal{L}_{D}^{(6)}+\mathcal{L}_{F}^{(6)}+\mathcal{L}_{\partial F}^{(6)}$, where

$$
\begin{aligned}
& \mathcal{L}_{D}^{(6)}= \frac{1}{2} c^{(6) \mu \alpha \beta \gamma} \bar{\psi} \gamma_{\mu} i D_{(\alpha} i D_{\beta} i D_{\gamma)} \psi \\
&+\frac{1}{2} d^{(6) \mu \alpha \beta \gamma} \bar{\psi} \gamma_{5} \gamma_{\mu} i D_{(\alpha} i D_{\beta} i D_{\gamma)} \psi \\
&+\frac{1}{2} e^{(6) \alpha \beta \gamma} \bar{\psi} i D_{(\alpha} i D_{\beta} i D_{\gamma} \psi \psi \\
&+\frac{1}{2} i f^{(6) \alpha \beta \gamma} \bar{\psi} \gamma_{5} i D_{(\alpha} i D_{\beta} i D_{\gamma)} \psi \\
&+\frac{1}{4} g^{(6) \mu \nu \alpha \beta \gamma} \bar{\psi} \sigma_{\mu \nu} i D_{(\alpha} i D_{\beta} i D_{\gamma)} \psi+\text { H.c. } \\
& \mathcal{L}_{F}^{(6)}= \frac{1}{4} c_{F}^{(6) \mu \alpha \beta \gamma} F_{\beta \gamma}\left(\bar{\psi} \gamma_{\mu} i D_{\alpha} \psi+\text { H.c. }\right) \\
&+\frac{1}{4} d_{F}^{(6) \mu \alpha \beta \gamma} F_{\beta \gamma}\left(\bar{\psi} \gamma_{5} \gamma_{\mu} i D_{\alpha} \psi+\text { H.c. }\right) \\
&+\frac{1}{4} e_{F}^{(6) \alpha \beta \gamma} F_{\beta \gamma}\left(\bar{\psi} i D_{\alpha} \psi+\text { H.c. }\right) \\
&+\frac{1}{4} i f_{F}^{(6) \alpha \beta \gamma} F_{\beta \gamma}\left(\bar{\psi} \gamma \gamma_{5} i D_{\alpha} \psi+\text { H.c. }\right) \\
&+\frac{1}{8} g_{F}^{(6) \mu \nu \alpha \beta \gamma} F_{\beta \gamma}\left(\bar{\psi} \sigma_{\mu \nu} i D_{\alpha} \psi+\text { H.c. }\right) \\
& \mathcal{L}_{\partial F}^{(6)}=- \frac{1}{2} m_{\partial F}^{(6) \alpha \beta \gamma} \partial_{\alpha} F_{\beta \gamma} \bar{\psi} \psi-\frac{1}{2} i m_{5 \partial F}^{(6) \alpha \beta \gamma} \partial_{\alpha} F_{\beta \gamma} \bar{\psi} \gamma \gamma_{5} \psi \\
&-\frac{1}{2} a_{\partial F}^{(6) \mu \alpha \beta \gamma} \partial_{\alpha} F_{\beta \gamma} \bar{\psi} \gamma_{\mu} \psi-\frac{1}{2} b_{\partial F}^{(6) \mu \alpha \beta \gamma} \partial_{\alpha} F_{\beta \gamma} \bar{\psi} \gamma_{5} \gamma_{\mu} \psi \\
&-\frac{1}{4} H_{\partial F}^{(6) \mu \nu \alpha \beta \gamma} \partial_{\alpha} F_{\beta \gamma} \bar{\psi} \sigma_{\mu \nu} \psi . \\
&(\mathrm{A} 6)
\end{aligned}
$$

In the above expressions, dimension superscripts for the minimal-SME coefficients are omitted. Coefficients with subscript $F$ or $\partial F$ are contracted with operators involving the electromagnetic field strength or its derivative, where indices $\mu, \nu$ are associated with spin properties, while $\alpha, \beta, \gamma$ are related to covariant momenta including field strengths. Parentheses on $n$ indices represent symmetrization with a factor of $1 / n !$. The properties of the coefficients for Lorentz violation appearing above are listed in Table I in Ref. [8].

\section{APPENDIX B: PERTURBATIVE ENERGY SHIFTS}

The perturbative energy shifts $\delta E_{n, \pm 1}^{w}$ due to Lorentz and $C P T$ violation for a fermion species $w$ of mass $m_{w}$ and charge $q=\sigma|q|$ in a magnetic field $\boldsymbol{B}=B \hat{x}_{3}$ in the apparatus frame can be obtained by applying perturbation calculations using Eq. (6). The analysis is performed with Lorentz- and $C P T$-violating operators appearing in $\mathcal{L}^{(3)}$, $\mathcal{L}^{(4)}, \mathcal{L}_{D}^{(5)}$, and $\mathcal{L}_{D}^{(6)}$, listed above in Appendix A. Following the discussion in Sec. III A, we find 


$$
\begin{aligned}
& \delta E_{n, \pm 1}^{w}=a_{w}^{0} \mp \sigma b_{w}^{3} \frac{m_{w}}{E_{n, \pm 1}^{w}} \mp \sigma H_{w}^{12}-c_{w}^{00} E_{n, \pm 1}^{w}-\left(c_{w}^{11}+c_{w}^{22}\right) \frac{(2 n+1 \mp \sigma)}{2 E_{n, \pm 1}^{w}}|q B| \pm \sigma d_{w}^{30} m_{w}-e_{w}^{0} m_{w} \\
& \mp \sigma\left(g_{w}^{012}-g_{w}^{021}\right) \frac{(2 n+1 \mp \sigma)}{2 E_{n, \pm 1}^{w}}|q B| \pm \sigma g_{w}^{120} E_{n, \pm 1}^{w}+a_{w}^{(5) 000}\left(E_{n, \pm 1}^{w}\right)^{2} \\
& +\left(a_{w}^{(5) 011}+a_{w}^{(5) 022}\right)\left(\frac{2 n+1 \mp \sigma}{2} \pm \sigma \frac{m_{w}}{2 E_{n, \pm 1}^{w}}\right)|q B|+\left(a_{w}^{(5) 101}+a_{w}^{(5) 202}\right)(2 n+1 \mp \sigma)|q B| \mp \sigma b_{w}^{(5) 300}{ }_{m} E_{n, \pm 1}^{w} \\
& \mp \sigma\left(b_{w}^{(5) 311}+b_{w}^{(5) 322}\right)\left(\frac{2 n+1 \mp \sigma}{2} \frac{m_{w}}{E_{n, \pm 1}^{w}} \pm \frac{1}{2} \sigma\right)|q B| \pm \sigma\left(H_{w}^{(5) 0102}-H_{w}^{(5) 0201}\right)(2 n+1 \mp \sigma)|q B| \\
& \mp \sigma H_{w}^{(5) 1200}\left(E_{n, \pm 1}^{w}\right)^{2} \mp \sigma\left(H_{w}^{(5) 1211}+H_{w}^{(5) 1222}\right)\left(\frac{2 n+1 \mp \sigma}{2} \pm \sigma \frac{m_{w}}{2 E_{n, \pm 1}^{w}}\right)|q B|+m_{w}^{(5) 00} m_{w} E_{n, \pm 1}^{w} \\
& +\left(m_{w}^{(5) 11}+m_{w}^{(5) 22}\right)\left(\frac{2 n+1 \mp \sigma}{2} \frac{m_{w}}{E_{n, \pm 1}^{w}} \pm \frac{1}{2} \sigma\right)|q B|-c_{w}^{(6) 0000}\left(E_{n, \pm 1}^{w}\right)^{3} \\
& -3\left(c_{w}^{(6) 0011}+c_{w}^{(6) 0022}\right)\left(\frac{2 n+1 \mp \sigma}{2} E_{n, \pm 1}^{w} \pm \frac{1}{2} \sigma m_{w}\right)|q B|-3\left(c_{w}^{(6) 1001}+c_{w}^{(6) 2002}\right) \frac{2 n+1 \mp \sigma}{2} E_{n, \pm 1}^{w}|q B| \\
& -3\left(c_{w}^{(6) 1111}+c_{w}^{(6) 2222}+c_{w}^{(6) 1122}+c_{w}^{(6) 2112}\right) \frac{(2 n+1 \mp \sigma)^{2}}{8 E_{n, \pm 1}^{w}}|q B|^{2} \pm \sigma d_{w}^{(6) 3000} m_{w}\left(E_{n, \pm 1}^{w}\right)^{2} \\
& +3\left(d_{w}^{(6) 3011}+d_{w}^{(6) 3022}\right)\left(\frac{2 n+1 \mp \sigma}{2} m_{w} \pm \frac{1}{2} \sigma E_{n, \pm 1}^{w}\right)|q B|-e_{w}^{(6) 000} m_{w}\left(E_{n, \pm 1}^{w}\right)^{2} \\
& -3\left(e_{w}^{(6) 011}+e_{w}^{(6) 022}\right)\left(\frac{2 n+1 \mp \sigma}{2} m_{w} \pm \frac{1}{2} \sigma E_{n, \pm 1}^{w}\right)|q B| \mp 3 \sigma\left(g_{w}^{(6) 01002}-g_{w}^{(6) 02001}\right) \frac{2 n+1 \mp \sigma}{2} E_{n, \pm 1}^{w}|q B| \\
& \mp 3 \sigma\left(g_{w}^{(6) 01112}+g_{w}^{(6) 01222}-g_{w}^{(6) 02122}-g_{w}^{(6) 02111}\right) \frac{(2 n+1 \mp \sigma)^{2}}{8 E_{n, \pm 1}^{w}}|q B|^{2} \pm \sigma g_{w}^{(6) 12000}\left(E_{n, \pm 1}^{w}\right)^{3} \\
& \pm 3 \sigma\left(g_{w}^{(6) 12011}+g_{w}^{(6) 12022}\right)\left(\frac{2 n+1 \mp \sigma}{2} E_{n, \pm 1}^{w} \pm \frac{1}{2} \sigma m_{w}\right)|q B|,
\end{aligned}
$$

where signs \pm denote the spin-up and spin-down states, respectively, and the unperturbed positive eigenenergies are given by $E_{n, \pm 1}^{w}=\sqrt{m_{w}^{2}+(2 n+1 \pm 1)|q B|}$. The additional energy shift contributions from $\mathcal{L}_{F}^{(5)}$ and $\mathcal{L}_{F}^{(6)}$ can be obtained by the substitutions (40) in Ref. [8], while $\mathcal{L}_{\partial F}^{(6)}$ has no energy shift contributions as $\partial_{\alpha} F_{\beta \gamma}=0$ for a uniform magnetic field in a Penning trap.

\section{APPENDIX C: TRANSFORMATIONS}

In this Appendix, we present the explicit relations between the coefficients for Lorentz violation $\tilde{b}_{w}^{\prime 3}, \tilde{c}_{w}^{11}+\tilde{c}_{w}^{22}$, $\tilde{b}_{w}^{311}+\tilde{b}_{w}^{322}, \tilde{b}_{w}^{\prime * 3}, \tilde{c}_{w}^{* 11}+\tilde{c}_{w}^{* 22}$, and $\tilde{b}_{w}^{* 311}+\tilde{b}_{w}^{* 322}$ in the apparatus frame and the constant ones in the Sun-centered frame.

For a Penning-trap experiment with a vertical upward magnetic field, applying transformation (22) with $R^{a j}$ being the identity matrix gives

$$
\begin{gathered}
\tilde{b}_{w}^{\prime 3}=\cos \omega_{\oplus} T_{\oplus} \tilde{b}_{w}^{\prime X} \sin \chi+\sin \omega_{\oplus} T_{\oplus} \tilde{b}_{w}^{\prime Y} \sin \chi+\tilde{b}_{w}^{\prime Z} \cos \chi, \\
\tilde{c}_{w}^{11}+\tilde{c}_{w}^{22}=\cos 2 \omega_{\oplus} T_{\oplus}\left(-\frac{1}{2}\left(\tilde{c}_{w}^{X X}-\tilde{c}_{w}^{Y Y}\right) \sin ^{2} \chi\right)+\sin 2 \omega_{\oplus} T_{\oplus}\left(-\tilde{c}_{w}^{(X Y)} \sin ^{2} \chi\right)+\cos \omega_{\oplus} T_{\oplus}\left(-\tilde{c}_{w}^{(X Z)} \sin 2 \chi\right) \\
+\sin \omega_{\oplus} T_{\oplus}\left(-\tilde{c}_{w}^{(Y Z)} \sin 2 \chi\right)+\frac{1}{4}\left(\tilde{c}_{w}^{X X}+\tilde{c}_{w}^{Y Y}\right)(3+\cos 2 \chi)+\tilde{c}_{w}^{Z Z} \sin ^{2} \chi,
\end{gathered}
$$




$$
\begin{aligned}
\tilde{b}_{w}^{311}+\tilde{b}_{w}^{322}= & \cos 3 \omega_{\oplus} T_{\oplus}\left(\left[-\frac{1}{4}\left(\tilde{b}_{w}^{X X X}-\tilde{b}_{w}^{X Y Y}\right)+\frac{1}{2} \tilde{b}_{w}^{Y(X Y)}\right] \sin ^{3} \chi\right)+\sin 3 \omega_{\oplus} T_{\oplus}\left(\left[-\frac{1}{2} \tilde{b}_{w}^{X(X Y)}-\frac{1}{4}\left(\tilde{b}_{w}^{Y X X}-\tilde{b}_{w}^{Y Y Y}\right)\right] \sin ^{3} \chi\right) \\
& +\cos 2 \omega_{\oplus} T_{\oplus}\left(\left[-\tilde{b}_{w}^{X(X Z)}+\tilde{b}_{w}^{Y(X Z)}-\frac{1}{2}\left(\tilde{b}_{w}^{Z X X}-\tilde{b}_{w}^{Z Y Y}\right)\right] \cos \chi \sin ^{2} \chi\right) \\
& +\sin 2 \omega_{\oplus} T_{\oplus}\left(\left[-\tilde{b}_{w}^{X(Y Z)}-\tilde{b}_{w}^{Y(X Z)}-\tilde{b}_{w}^{Z(X Y)}\right] \cos \chi \sin ^{2} \chi\right) \\
& +\cos \omega_{\oplus} T_{\oplus}\left(\frac{1}{8} \tilde{b}_{w}^{X X X}(5+3 \cos 2 \chi) \sin \chi+\frac{1}{8} \tilde{b}_{w}^{X Y Y}(7+\cos 2 \chi) \sin \chi+\tilde{b}_{w}^{X Z Z} \sin ^{3} \chi\right. \\
& \left.-\frac{1}{2} \tilde{b}_{w}^{Y(X Y)} \sin ^{3} \chi-2 \tilde{b}_{w}^{Z(X Z)} \cos ^{2} \chi \sin \chi\right) \\
& +\sin \omega_{\oplus} T_{\oplus}\left(-\frac{1}{2} \tilde{b}_{w}^{X(X Y)} \sin ^{3} \chi+\frac{1}{8} \tilde{b}_{w}^{Y X X}(7+\cos 2 \chi) \sin \chi+\frac{1}{8} \tilde{b}_{w}^{Y Y Y}(5+3 \cos 2 \chi) \sin \chi\right. \\
& \left.+\tilde{b}_{w}^{Y Z Z} \sin ^{3} \chi-2 \tilde{b}_{w}^{Z(Y Z)} \cos ^{2} \chi \sin \chi\right) \\
& -\left(\tilde{b}_{w}^{X(X Z)}+\tilde{b}_{w}^{Y(X Z)}-\tilde{b}_{w}^{Z Z Z}\right) \cos \chi \sin ^{2} \chi+\left(\tilde{b}_{w}^{Z X X}+\tilde{b}_{w}^{Z Y Y}\right) \cos \chi \cos 2 \chi .
\end{aligned}
$$

In the case where the trap uses a horizontal magnetic field with an angle $\theta$ from the local south in the counterclockwise direction, the corresponding Euler angles relating the apparatus frame to the standard laboratory frame discussed in Sec. III C are found to be $(\alpha, \beta, \gamma)=(\theta, \pi / 2,0)$. Substituting this to the matrix (21) and applying the transformation (22) give the following relations:

$$
\begin{aligned}
& \tilde{b}_{w}^{\prime 3}=\cos \omega_{\oplus} T_{\oplus}\left(\tilde{b}_{w}^{\prime X} \cos \theta \cos \chi+\tilde{b}_{w}^{\prime Y} \sin \theta\right)+\sin \omega_{\oplus} T_{\oplus}\left(-\tilde{b}_{w}^{\prime X} \sin \theta+\tilde{b}_{w}^{\prime Y} \cos \theta \cos \chi\right)-\tilde{b}_{w}^{\prime Z} \cos \theta \sin \chi, \\
& \tilde{c}_{w}^{11}+\tilde{c}_{w}^{22}=\cos 2 \omega_{\oplus} T_{\oplus}\left(\frac{1}{8}\left(\tilde{c}_{w}^{X X}-\tilde{c}_{w}^{Y Y}\right)\left(1-3 \cos 2 \theta-2 \cos ^{2} \theta \cos 2 \chi\right)-\tilde{c}_{w}^{(X Y)} \cos \chi \sin 2 \theta\right) \\
& +\sin 2 \omega_{\oplus} T_{\oplus}\left(\frac{1}{2}\left(\tilde{c}_{w}^{X X}-\tilde{c}_{w}^{Y Y}\right) \cos \chi \sin 2 \theta+\frac{1}{4} \tilde{c}_{w}^{(X Y)}\left(1-3 \cos 2 \theta-2 \cos ^{2} \theta \cos 2 \chi\right)\right) \\
& +\cos \omega_{\oplus} T_{\oplus}\left(\tilde{c}_{w}^{(X Z)} \cos ^{2} \theta \sin 2 \chi+\tilde{c}_{w}^{(Y Z)} \sin 2 \theta \sin \chi\right)+\sin \omega_{\oplus} T_{\oplus}\left(-\tilde{c}_{w}^{(X Z)} \sin 2 \theta \sin \chi+\tilde{c}_{w}^{(Y Z)} \cos ^{2} \theta \sin 2 \chi\right) \\
& +\frac{1}{2}\left(\tilde{c}_{w}^{X X}+\tilde{c}_{w}^{Y Y}\right)\left(\cos ^{2} \theta+\cos ^{2} \chi \sin ^{2} \theta+\sin ^{2} \chi\right)+\tilde{c}_{w}^{Z Z}\left(\cos ^{2} \chi+\sin ^{2} \theta \sin ^{2} \chi\right), \\
& \tilde{b}_{w}^{311}+\tilde{b}_{w}^{322}=\cos 3 \omega_{\oplus} T_{\oplus}\left(\left[\frac{1}{64}\left(\tilde{b}_{w}^{X X X}-\tilde{b}_{w}^{X Y Y}\right)-\frac{1}{32} \tilde{b}_{w}^{Y(X Y)}\right]\left[3(\cos \theta-5 \cos 3 \theta) \cos \chi-4 \cos ^{3} \theta \cos 3 \chi\right]\right. \\
& \left.+\left[\frac{1}{16} \tilde{b}_{w}^{X(X Y)}+\frac{1}{32}\left(\tilde{b}_{w}^{Y X X}-\tilde{b}_{w}^{Y Y Y}\right)\right]\left[3 \sin \theta\left(1-4 \cos ^{2} \theta \cos 2 \chi\right)-5 \sin 3 \theta\right]\right) \\
& +\sin 3 \omega_{\oplus} T_{\oplus}\left(\left[-\frac{1}{32}\left(\tilde{b}_{w}^{X X X}-\tilde{b}_{w}^{X Y Y}\right)+\frac{1}{16} \tilde{b}_{w}^{Y(X Y)}\right]\left[3 \sin \theta\left(1-4 \cos ^{2} \theta \cos 2 \chi\right)-5 \sin 3 \theta\right]\right. \\
& \left.+\left[\frac{1}{32} \tilde{b}_{w}^{X(X Y)}+\frac{1}{64}\left(\tilde{b}_{w}^{Y X X}-\tilde{b}_{w}^{Y Y Y}\right)\right]\left[3(\cos \theta-5 \cos 3 \theta) \cos \chi-4 \cos ^{3} \theta \cos 3 \chi\right]\right) \\
& +\cos 2 \omega_{\oplus} T_{\oplus}\left(\left[-\frac{1}{16} \tilde{b}_{w}^{X(X Z)}+\frac{1}{16} \tilde{b}_{w}^{Y(X Z)}-\frac{1}{32}\left(\tilde{b}_{w}^{Z X X}-\tilde{b}_{w}^{Z Y Y}\right)\right]\left[(\cos \theta-5 \cos 3 \theta) \sin \chi-4 \cos ^{3} \theta \sin 3 \chi\right]\right. \\
& \left.+\left(\tilde{b}_{w}^{X(Y Z)}+\tilde{b}_{w}^{Y(X Z)}+\tilde{b}_{w}^{Z(X Y)}\right) \sin \theta \cos ^{2} \theta \sin 2 \chi\right) \\
& +\sin 2 \omega_{\oplus} T_{\oplus}\left(\left[-\tilde{b}_{w}^{X(X Z)}+\tilde{b}_{w}^{Y(X Z)}-\frac{1}{2}\left(\tilde{b}_{w}^{Z X X}-\tilde{b}_{w}^{Z Y Y}\right)\right] \sin \theta \cos ^{2} \theta \sin 2 \chi\right. \\
& \left.-\left(\frac{1}{16} \tilde{b}_{w}^{X(Y Z)}+\frac{1}{16} \tilde{b}_{w}^{Y(X Z)}+\frac{1}{16} \tilde{b}_{w}^{Z(X Y)}\right)\left[(\cos \theta-5 \cos 3 \theta) \sin \chi-4 \cos ^{3} \theta \sin 3 \chi\right]\right)
\end{aligned}
$$




$$
\begin{aligned}
& +\cos \omega_{\oplus} T_{\oplus} \\
& \times\left(\frac{1}{16} \tilde{b}_{w}^{X X X} \cos \theta \cos \chi\left(-6 \cos ^{2} \theta \cos 2 \chi+3 \cos 2 \theta+7\right)-\frac{1}{2} \tilde{b}_{w}^{X(X Y)} \sin \theta\left(\cos ^{2} \theta \cos 2 \chi+\sin ^{2} \theta \cos ^{2} \chi+\sin ^{2} \chi\right)\right. \\
& +\frac{1}{16} \tilde{b}_{w}^{X Y Y} \cos \theta \cos \chi\left(-2 \cos ^{2} \theta \cos 2 \chi+\cos 2 \theta+13\right)+\tilde{b}_{w}^{X Z Z} \cos \theta \cos \chi\left(\sin ^{2} \theta \sin ^{2} \chi+\cos ^{2} \chi\right) \\
& +\frac{1}{32} \tilde{b}_{w}^{Y X X}\left[\sin \theta\left(25-4 \cos ^{2} \theta \cos 2 \chi\right)+\sin 3 \theta\right]+\frac{1}{16} \tilde{b}_{w}^{Y(X Y)} \cos \theta\left[(\cos 2 \theta-7) \cos \chi-2 \cos ^{2} \theta \cos 3 \chi\right] \\
& +\frac{1}{32} \tilde{b}_{w}^{Y Y Y}\left[\sin \theta\left(11-12 \cos ^{2} \theta \cos 2 \chi\right)+3 \sin 3 \theta\right]+\tilde{b}_{w}^{Y Z Z} \sin \theta\left(\sin ^{2} \theta \sin ^{2} \chi+\cos ^{2} \chi\right) \\
& \left.-2 \tilde{b}_{w}^{Z(X Z)} \cos ^{3} \theta \sin ^{2} \chi \cos \chi-2 \tilde{b}_{w}^{Z(Y Z)} \sin \theta \cos ^{2} \theta \sin ^{2} \chi\right) \\
& +\sin \omega_{\oplus} T_{\oplus} \\
& \times\left(\frac{1}{32} \tilde{b}_{w}^{X X X}\left[\sin \theta\left(12 \cos ^{2} \theta \cos 2 \chi-11\right)-3 \sin 3 \theta\right]+\frac{1}{16} \tilde{b}_{w}^{X(X Y)} \cos \theta\left[(\cos 2 \theta-7) \cos \chi-2 \cos ^{2} \theta \cos 3 \chi\right]\right. \\
& +\frac{1}{32} \tilde{b}_{w}^{X Y Y}\left[\sin \theta\left(4 \cos ^{2} \theta \cos 2 \chi-25\right)-\sin 3 \theta\right]-\tilde{b}_{w}^{X Z Z} \sin \theta\left(\sin ^{2} \theta \sin ^{2} \chi+\cos ^{2} \chi\right) \\
& +\frac{1}{16} \tilde{b}_{w}^{Y X X} \cos \theta \cos \chi\left(-2 \cos ^{2} \theta \cos 2 \chi+\cos 2 \theta+13\right)+\frac{1}{2} \tilde{b}_{w}^{Y(X Y)} \sin \theta\left(\cos ^{2} \theta \cos 2 \chi+\sin ^{2} \theta \cos ^{2} \chi+\sin ^{2} \chi\right) \\
& +\frac{1}{16} \tilde{b}_{w}^{Y Y Y} \cos \theta \cos \chi\left(-6 \cos ^{2} \theta \cos 2 \chi+3 \cos 2 \theta+7\right)+\tilde{b}_{w}^{Y Z Z} \cos \theta \cos \chi\left(\sin ^{2} \theta \sin ^{2} \chi+\cos ^{2} \chi\right) \\
& \left.+2 \tilde{b}_{w}^{Z(X Z)} \sin \theta \cos ^{2} \theta \sin ^{2} \chi-2 \tilde{b}_{w}^{Z(Y Z)} \cos ^{3} \theta \sin ^{2} \chi \cos \chi\right) \\
& +\frac{1}{8}\left(\tilde{b}_{w}^{X(X Z)}+\tilde{b}_{w}^{Y(X Z)}-\tilde{b}_{w}^{Z Z Z}\right) \cos \theta\left(-4 \cos 2 \theta \sin ^{3} \chi+5 \sin \chi+\sin 3 \chi\right) \\
& +\frac{1}{16}\left(\tilde{b}_{w}^{Z X X}+\tilde{b}_{w}^{Z Y Y}\right)\left[2 \cos ^{3} \theta \sin 3 \chi-\cos \theta \sin \chi(3 \cos 2 \theta+11)\right] .
\end{aligned}
$$

The corresponding transformation results for the starred tilde quantities $\tilde{b}_{w}^{\prime * 3}, \tilde{c}_{w}^{* 11}+\tilde{c}_{w}^{* 22}$, and $\tilde{b}_{w}^{* 311}+\tilde{b}_{w}^{* 322}$ have the same form as these given above.

[1] V. A. Kostelecký and S. Samuel, Phys. Rev. D 39, 683 (1989); V. A. Kostelecký and R. Potting, Nucl. Phys. B359, 545 (1991); Phys. Rev. D 51, 3923 (1995).

[2] D. Colladay and V. A. Kostelecký, Phys. Rev. D 55, 6760 (1997); 58, 116002 (1998).

[3] O. W. Greenberg, Phys. Rev. Lett. 89, 231602 (2002).

[4] V. A. Kostelecký and N. Russell, Rev. Mod. Phys. 83, 11 (2011); 2020 edition arXiv:0801.0287v13.

[5] S. Ulmer et al., Nature (London) 524, 196 (2015).

[6] R. S. Van Dyck, Jr., P. B. Schwinberg, and H. G. Dehmelt, Phys. Rev. Lett. 59, 26 (1987); Phys. Rev. D 34, 722 (1986).

[7] C. Smorra et al., Nature (London) 550, 371 (2017).

[8] Y. Ding and V. A. Kostelecký, Phys. Rev. D 94, 056008 (2016).

[9] V. A. Kostelecký, Phys. Rev. D 69, 105009 (2004).
[10] H. Dehmelt, R. Mittleman, R. S. Van Dyck, Jr., and P. Schwinberg, Phys. Rev. Lett. 83, 4694 (1999).

[11] G. Schneider et al., Science 358, 1081 (2017).

[12] G. Gabrielse, A. Khabbaz, D. S. Hall, C. Heimann, H. Kalinowsky, and W. Jhe, Phys. Rev. Lett. 82, 3198 (1999).

[13] R. Bluhm, V. A. Kostelecký, and N. Russell, Phys. Rev. Lett. 79, 1432 (1997).

[14] R. Bluhm, V. A. Kostelecký, and N. Russell, Phys. Rev. D 57, 3932 (1998).

[15] R. K. Mittleman, I. I. Ioannou, H. G. Dehmelt, and N. Russell, Phys. Rev. Lett. 83, 2116 (1999).

[16] Y. Ding, Symmetry 11, 1220 (2019).

[17] C. Smorra et al., Nature (London) 575, 310 (2019).

[18] M. Hayakawa, Phys. Lett. B 478, 394 (2000).

[19] S. M. Carroll, J. A. Harvey, V. A. Kostelecký, C. D. Lane, and T. Okamoto, Phys. Rev. Lett. 87, 141601 (2001). 
[20] A. C. Lehum, Europhys. Lett. 112, 51001 (2015); M. Faizal and P. A. Ganai, Europhys. Lett. 111, 21001 (2015); H. Belich, L. D. Bernald, P. Gaete, J. A. Helayël-Neto, and F. J. L. Leal, Eur. Phys. J. C 75, 291 (2015); J. L. Chkareuli, Bled Workshops Phys. 15, 46 (2014); A. C. Lehum, J. R. Nascimento, A. Yu. Petrov, and A. J. da Silva, Phys. Rev. D 88, 045022 (2013); H. Belich, L. D. Bernald, P. Gaete, and J. A. Helayël-Neto, Eur. Phys. J. C 73, 2632 (2013); A. B. Clark, J. High Energy Phys. 01 (2014) 134; C. F. Farias, A. C. Lehum, J. R. Nascimento, and A. Yu. Petrov, Phys. Rev. D 86, 065035 (2012); D. Redigolo, Phys. Rev. D 85, 085009 (2012); D. Colladay and P. McDonald, Phys. Rev. D 83, 025021 (2011); P. A. Bolokhov, S. G. Nibbelink, and M. Pospelov, Phys. Rev. D 72, 015013 (2005); M. S. Berger and V. A. Kostelecký, Phys. Rev. D 65, 091701(R) (2002).

[21] M. Cambiaso, R. Lehnert, and R. Potting, Phys. Rev. D 90, 065003 (2014); I. T. Drummond, Phys. Rev. D 88, 025009 (2013); M. A. Hohensee, D. F. Phillips, and R. L. Walsworth, arXiv:1210.2683; M. Schreck, Phys. Rev. D 86, 065038 (2012); F. R. Klinkhamer and M. Schreck, Nucl. Phys. B848, 90 (2011); C. M. Reyes, Phys. Rev. D 87, 125028 (2013); 82, 125036 (2010); V. A. Kostelecký and R. Lehnert, Phys. Rev. D 63, 065008 (2001).

[22] J. E. G. Silva, arXiv:1602.07345; J. E. G. Silva, R. V. Maluf, and C. A. S. Almeida, Phys. Lett. B 766, 263 (2017); J. Foster and R. Lehnert, Phys. Lett. B 746, 164 (2015); N. Russell, Phys. Rev. D 91, 045008 (2015); M. Schreck, Phys. Rev. D 91, 105001 (2015); 92, 125032 (2015); Eur. J. Phys. C 75, 187 (2015); Phys. Rev. D 93, 105017 (2016); 94, 025019 (2016); J. E. G. Silva and C. A. S. Almeida, Phys. Lett. B 731, 74 (2014); V. A. Kostelecký, N. Russell, and R. Tso, Phys. Lett. B 716, 470 (2012); D. Colladay and P. McDonald, Phys. Rev. D 85, 044042 (2012); 92, 085031 (2015); V. A. Kostelecký, Phys. Lett. B 701, 137 (2011); B. Edwards and V. A. Kostelecký, Phys. Lett. B 786, 319 (2018).

[23] R. Bluhm, V. A. Kostelecký, and C. D. Lane, Phys. Rev. Lett. 84, 1098 (2000); M. Deile et al., arXiv:hep-ex/0110044; G. W. Bennett et al., Phys. Rev. Lett. 100, 091602 (2008); Y. V. Stadnik, B. M. Roberts, and V. V. Flambaum, Phys. Rev. D 90, 045035 (2014).

[24] A. H. Gomes, V. A. Kostelecký, and A. J. Vargas, Phys. Rev. D 90, 076009 (2014).

[25] V. A. Kostelecký and A. J. Vargas, Phys. Rev. D 92, 056002 (2015).

[26] V. A. Kostelecký and A. J. Vargas, Phys. Rev. D 98, 036003 (2018).
[27] V. A. Kostelecký and M. Mewes, Phys. Rev. D 88, 096006 (2013).

[28] V. A. Kostelecký and Z. Li, Phys. Rev. D 99, 056016 (2019).

[29] V. A. Kostelecký and M. Mewes, Phys. Rev. D 80, 015020 (2009); Astrophys. J. 689, L1 (2008).

[30] V. A. Kostelecký and M. Mewes, Phys. Rev. D 85, 096005 (2012); J. S. Díaz, V. A. Kostelecký, and M. Mewes, Phys. Rev. D 89, 043005 (2014).

[31] V. A. Kostelecký and M. Mewes, Phys. Lett. B 757, 510 (2016); Q. G. Bailey, V. A. Kostelecký, and R. Xu, Phys. Rev. D 91, 022006 (2015); V. A. Kostelecký and J. D. Tasson, Phys. Lett. B 749, 551 (2015).

[32] The antisymmetry of the index pair " 12 " on the right sides of the definitions ((12) and (13) is reflected by the spin index pair $\mu \nu$ of the relevant coefficients together with the form of the expression. For example, the definition of $\tilde{b}_{w}^{\prime 3}$ in (12) contains a term of $g_{w}^{120}-g_{w}^{012}+g_{w}^{021} \cdot g_{w}^{120}$ is antisymmetric on the index pair " 12 " from the property of the $g_{w}^{\mu \nu \alpha}$ coefficient as the first two indices are contracted with the antisymmetric Dirac matrix $\sigma_{\mu \nu}$. The remaining expression $-g_{w}^{012}+g_{w}^{021}$ is antisymmetric on the index pair " 12 " by observation. Similar arguments can be made for other terms containing the index pair " 12 " to prove that it is antisymmetric in these definitions.

[33] V. A. Kostelecký and C. D. Lane, Phys. Rev. D 60, 116010 (1999).

[34] V. A. Kostelecký and M. Mewes, Phys. Rev. D 66, 056005 (2002).

[35] The common adopted " $y$-convention" for the Euler angles $(\alpha, \beta, \gamma)$ from coordinate system $(x, y, z)$ to $\left(x^{1}, x^{2}, x^{3}\right)$ is defined as follows. First, rotate $x y z$ counterclockwise around its $\hat{z}$ axis by $\alpha$ to give $x^{\prime} y^{\prime} z^{\prime}$. Then, rotate $x^{\prime} y^{\prime} z^{\prime}$ counterclockwise around its $\hat{y}$ axis by $\beta$ to give $x^{\prime \prime} y^{\prime \prime} z^{\prime \prime}$. Finally, rotate $x^{\prime \prime} y^{\prime \prime} z^{\prime \prime}$ counterclockwise around its $\hat{z}^{\prime \prime}$ axis by $\gamma$ to give $x^{1} x^{2} x^{3}$

[36] F. Canè, D. Bear, D. F. Phillips, M. S. Rosen, C. L. Smallwood, R. E. Stoner, R. L. Walsworth, and V. A. Kostelecký, Phys. Rev. Lett. 93, 230801 (2004).

[37] B. R. Heckel, E. G. Adelberger, C. E. Cramer, T. S. Cook, S. Schlamminger, and U. Schmidt, Phys. Rev. D 78, 092006 (2008).

[38] R. Bluhm, V. A. Kostelecký, C. D. Lane, and N. Russell, Phys. Rev. D 68, 125008 (2003); Phys. Rev. Lett. 88, 090801 (2002). 